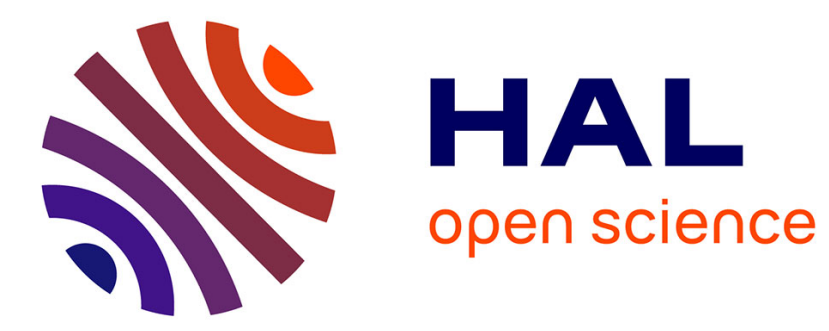

\title{
Effects of an axial flow on the centrifugal, elliptic and hyperbolic instabilities in Stuart vortices
}

Manikandan Mathur, Sabine Ortiz, Thomas Dubos, Jean-Marc Chomaz

\section{To cite this version:}

Manikandan Mathur, Sabine Ortiz, Thomas Dubos, Jean-Marc Chomaz. Effects of an axial flow on the centrifugal, elliptic and hyperbolic instabilities in Stuart vortices. Journal of Fluid Mechanics, 2014, 758 (november), pp.565 - 585. 10.1017/jfm.2014.534 . hal-01117122

\section{HAL Id: hal-01117122}

https://hal-polytechnique.archives-ouvertes.fr/hal-01117122

Submitted on 16 Feb 2015

HAL is a multi-disciplinary open access archive for the deposit and dissemination of scientific research documents, whether they are published or not. The documents may come from teaching and research institutions in France or abroad, or from public or private research centers.
L'archive ouverte pluridisciplinaire HAL, est destinée au dépôt et à la diffusion de documents scientifiques de niveau recherche, publiés ou non, émanant des établissements d'enseignement et de recherche français ou étrangers, des laboratoires publics ou privés. 


\title{
Effects of an axial flow on the centrifugal, elliptic and hyperbolic instabilities in Stuart vortices
}

\author{
Manikandan Mathur ${ }^{1, \dagger}$, Sabine Ortiz ${ }^{2,3}$, Thomas Dubos ${ }^{4}$ and \\ Jean-Marc Chomaz ${ }^{2}$ \\ ${ }^{1}$ Department of Aerospace Engineering, Indian Institute of Technology Madras, Chennai 600036, India \\ ${ }^{2}$ LadHyX, CNRS-École Polytechnique, F-91128 Palaiseau CEDEX, France \\ ${ }^{3}$ UME/DFA, ENSTA, Chemin de la Hunière, 91761 Palaiseau CEDEX, France \\ ${ }^{4}$ Laboratoire de Météorologie Dynamique/IPSL, École Polytechnique, Palaiseau, France
}

(Received 24 January 2014; revised 11 June 2014; accepted 9 September 2014; first published online 10 October 2014)

Linear stability of the Stuart vortices in the presence of an axial flow is studied. The local stability equations derived by Lifschitz \& Hameiri (Phys. Fluids A, vol. 3 (11), 1991, pp. 2644-2651) are rewritten for a three-component (3C) two-dimensional (2D) base flow represented by a $2 \mathrm{D}$ streamfunction and an axial velocity that is a function of the streamfunction. We show that the local perturbations that describe an eigenmode of the flow should have wavevectors that are periodic upon their evolution around helical flow trajectories that are themselves periodic once projected on a plane perpendicular to the axial direction. Integrating the amplitude equations around periodic trajectories for wavevectors that are also periodic, it is found that the elliptic and hyperbolic instabilities, which are present without the axial velocity, disappear beyond a threshold value for the axial velocity strength. Furthermore, a threshold axial velocity strength, above which a new centrifugal instability branch is present, is identified. A heuristic criterion, which reduces to the Leibovich \& Stewartson criterion in the limit of an axisymmetric vortex, for centrifugal instability in a non-axisymmetric vortex with an axial flow is then proposed. The new criterion, upon comparison with the numerical solutions of the local stability equations, is shown to describe the onset of centrifugal instability (and the corresponding growth rate) very accurately.

Key words: vortex flows, vortex instability

\section{Introduction}

Stability analyses of vortical flows provide significant insights into understanding various fluid phenomena (Saffman 1992). For example, the linear instability of two-dimensional (2D) vortices often results in complex three-dimensional (3D) vortex structures, found in abundance in turbulent flows (Saffman 1992; Kerswell 2002). In this paper, we focus our study on $2 \mathrm{D}$ vortices with an axial flow, a situation

$†$ Email address for correspondence: manims@ae.iitm.ac.in 
prevalent in physical settings such as tornadoes, airplane leading-edge and trailing vortices, swirling flow in combustion and vortex streets with an axial flow.

Motivated by vortex breakdown observations (Hall 1972; Leibovich 1978), Leibovich \& Stewartson (1983) performed an asymptotic normal mode analysis to derive a sufficient condition for an inviscid instability of a steady axisymmetric vortex with an axial flow. Further experimental studies on swirling jets, which can also be thought of as vortices with an axial flow, showed that a double-helix structure appears for $0.6<S<1$, followed by vortex breakdown beyond $S \approx 1.3$ (Billant, Chomaz \& Huerre 1998). Here, $S$ is the swirl parameter that measures the relative strength of the swirl velocity with respect to the axial velocity. Gallaire \& Chomaz (2003) performed a numerical global stability analysis of axisymmetric vortices (with circulation decaying to zero far away from the core) with realistic axial and azimuthal velocity profiles to identify the mechanisms leading to the appearance of the double-helix structures before breakdown. Other experimental evidence (Gallaire, Rott \& Chomaz 2004; Liang \& Maxworthy 2005; Oberleithner et al. 2011), normal mode and global stability analyses (Loiseleux, Chomaz \& Huerre 1998; Gallaire et al. 2006; Healey 2008; Oberleithner et al. 2011) have further shown the prevalence of absolute instability and vortex breakdown in swirling jets. In a related study, Lacaze, Birbaud \& Le Dizès (2005) added a small strain to the axisymmetric Rankine vortex with an axial flow and performed a normal mode analysis using asymptotic methods to demonstrate that the most unstable mode of the elliptic instability is modified by an axial flow. Axial flow is also an important factor for the secondary instability of the Ekman layer rolls (Dubos, Barthlott \& Drobinski 2008). In the current study, instead of the normal mode and global analysis, we employ the local stability approach (Lifschitz \& Hameiri 1991).

The local stability approach, a theory based on the Wentzel-Kramers-BrillouinJeffreys (WKBJ) approximation (Bender \& Orszag 1999), investigates inviscid, 3D, short-wavelength instabilities that develop on specific fluid trajectories in various base flows (Lifschitz \& Hameiri 1991). Though applicable to arbitrary 3D base flows, the local approach has so far mostly been used for either 2D base flows (Godeferd, Cambon \& Leblanc 2001) or axisymmetric 3D base flows (Lifschitz, Suters \& Beale 1996; Hattori \& Fukumoto 2003; Hattori \& Hijiya 2010), with a focus on the growth of specific disturbances on periodic trajectories leading to centrifugal, elliptic and hyperbolic instabilities. Recently, Hattori \& Fukumoto (2012) performed a local stability analysis to study the effects of axial flow on the so-called curvature instability of a helical vortex tube for which the angular velocity is constant up to the first order of a small parameter; elliptic instability was not considered in this study.

Eckhoff \& Storesletten (1978) employed the local approach to investigate the effects of an axial flow on an axisymmetric vortex in a compressible flow, and in a follow-up paper, Eckhoff (1984) showed that the results from the local approach agreed with the asymptotic analysis of Leibovich \& Stewartson (1983) in the limit of incompressibility. Le Duc \& Leblanc (1999) and Leblanc \& Le Duc (2005) have further extended the study of axisymmetric vortices with an axial flow to establish the connection between the local approach and the high-wavenumber asymptotic limits of the normal mode approach. The local approach has also provided significant insights into the elliptic (Bayly 1986; Bayly, Holm \& Lifschitz 1996; Le Dizès \& Eloy 1999) and hyperbolic (Friedlander \& Vishik 1991; Leblanc 1997) instabilities in various 2D flows. Hattori \& Hijiya (2010) studied the effects of an axial flow on the instability of Hill's vortex, an axisymmetric flow with dependence on $r$ and $z$ in cylindrical polar coordinates; the 
authors find a stabilizing effect for small axial flows, followed by the emergence of a region of centrifugal instability for large axial flows.

In the present study, we investigate the stability of Stuart vortices, which model mixing-layer vortices (Stuart 1967). The centrifugal, elliptic and hyperblolic instabilities in the Stuart vortices with and without background rotation were studied using the local stability approach by Godeferd et al. (2001), one of the main results being that anticyclonic rotation destabilizes the vortices. In this paper, expressing the general local stability equations (Lifschitz \& Hameiri 1991) for base flows with non-zero velocity components in all three Cartesian directions $(x, y, z)$, but with dependence only on $(x, y)$, the so-called three-component $2 \mathrm{D}$ base flows (3C2D), we investigate how an axial flow modifies the 3D stability characteristics of Stuart vortices.

In $\S 2$, we present details of the theory and implementation of the local stability approach to investigate $2 \mathrm{D}$ flows with an axial flow. In $\S 3$, we show the results of a systematic study of the effects of an axial flow on the stability of Stuart vortices. We then discuss our results in $\S 4$, and conclude in $\S 5$.

\section{Theory and methods}

We consider inviscid, incompressible, steady 3C2D base flows with a velocity field $(u(x, y), v(x, y), w(x, y))$ in a $\boldsymbol{x}=(x, y, z)$ Cartesian coordinate system. The flow being incompressible, the velocity field $(u, v)$ on the $x y$-plane is represented by a streamfunction $\psi(x, y)$ such that $u=-\partial \psi / \partial y$ and $v=\partial \psi / \partial x$. For a steady base flow with no dependence on one of the coordinates ( $z$ in our scenario), solutions of the Euler equations satisfy $w=f(\psi)$, where $f$ is any function of the streamfunction $\psi$; $f(\psi)$ is assumed smooth for compatibility with the viscous case. Hence, we have:

$$
\frac{\partial w}{\partial x}=f^{\prime}(\psi) \frac{\partial \psi}{\partial x}, \quad \frac{\partial w}{\partial y}=f^{\prime}(\psi) \frac{\partial \psi}{\partial y} .
$$

For vortical base flows, the fluid trajectories are then helices and their projections on the $x y$-plane are closed curves (and hence periodic), as shown in figure 1.

\subsection{WKB theory}

Within the WKBJ approximation, perturbations in velocity and pressure take the form:

$$
\begin{aligned}
& \boldsymbol{u}^{\prime}=\exp (\mathrm{i} \phi(\boldsymbol{x}, t) / \epsilon)\left[\boldsymbol{a}(\boldsymbol{x}, t)+\epsilon \boldsymbol{a}_{\epsilon}(\boldsymbol{x}, t)+\cdots\right], \\
& p^{\prime}=\exp (\mathrm{i} \phi(\boldsymbol{x}, t) / \epsilon)\left[\pi(\boldsymbol{x}, t)+\epsilon \pi_{\epsilon}(\boldsymbol{x}, t)+\cdots\right],
\end{aligned}
$$

respectively. The scalar function $\phi(x, t)$ is assumed real. Assuming $\epsilon \ll 1$, the continuity and momentum equations, retaining only the $O\left(\epsilon^{-1}\right)$ and $O\left(\epsilon^{0}\right)$ terms, reduce to Lifschitz \& Hameiri (1991):

$$
\begin{gathered}
\boldsymbol{a} \cdot \boldsymbol{k}=0, \\
\frac{\mathrm{d} \boldsymbol{k}}{\mathrm{d} t}=-(\nabla \boldsymbol{U})^{\mathrm{T}} \cdot \boldsymbol{k}, \\
\frac{\mathrm{d} \boldsymbol{a}}{\mathrm{d} t}=-\nabla \boldsymbol{U} \cdot \boldsymbol{a}+\frac{2}{|\boldsymbol{k}|^{2}}[(\nabla \boldsymbol{U} \cdot \boldsymbol{a}) \cdot \boldsymbol{k}] \boldsymbol{k},
\end{gathered}
$$




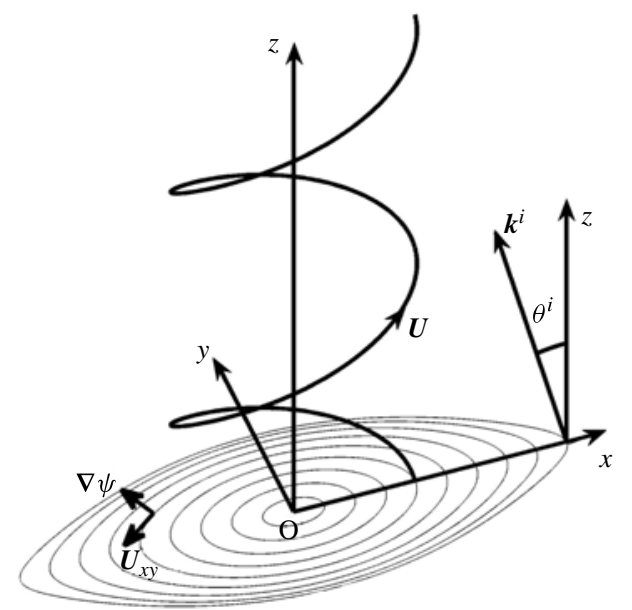

FIGURE 1. A depiction of the vortical base flow we consider, with one sample helical fluid trajectory. Projections of several trajectories on the $x y$-plane are also shown. On the 2D projection of a 3D helical trajectory, the local 2D Serret-Frenet frame is based on $\nabla \psi$ and $\boldsymbol{U}_{x y}$, which are normal and tangent to the projected trajectory, respectively. The initial wavevector $\boldsymbol{k}^{i}$ makes an angle $\theta^{i}$ with the $z$-axis.

where $\mathrm{d} / \mathrm{d} t(=\partial / \partial t+\boldsymbol{U} \cdot \nabla)$ is the total time derivative with respect to the base flow $\boldsymbol{U}=u \boldsymbol{e}_{x}+v \boldsymbol{e}_{y}+w \boldsymbol{e}_{z}=(-\partial \psi / \partial y) \boldsymbol{e}_{x}+(\partial \psi / \partial x) \boldsymbol{e}_{y}+w \boldsymbol{e}_{z}=\boldsymbol{U}_{x y}+w \boldsymbol{e}_{z}$, and $\boldsymbol{k}=\nabla \phi / \epsilon$ is the wavevector. For 3C2D flows, (2.6) gives $\mathrm{d} \boldsymbol{a} / \mathrm{d} t=0$ for all $\boldsymbol{a}$ that are aligned with $\boldsymbol{e}_{z}$. The transformation $\boldsymbol{k}=k_{0} \boldsymbol{k}$ (for any constant $k_{0}$ ) leaves the (2.4)-(2.6) unchanged; this scale invariance property is not trivial since the (2.4)-(2.6) are not homogeneous in $x$ and $y$. An important consequence of the scale invariance property is that the growth rate is the same at all spatial scales.

Equations (2.5) and (2.6) are transport equations along 3D fluid trajectories in the base flow, describing the evolution of any initial small-scale perturbation in the limit $\epsilon \ll 1$. Since the right-hand sides of (2.5) and (2.6) depend only on $\boldsymbol{a}$ and $\boldsymbol{k}$, and not their derivatives or integrals, the equations may be integrated independently on each 3D trajectory - that is, in the present case, a non-circular helix (figure 1), i.e. a winding around a non-circular cylinder.

In the normal mode approach, the temporal stability of a 3C2D base flow corresponds to the global eigenvalue problem of determining the (eigen)frequency $\omega$ and eigenfunction $F(x, y)$ as a function of $k_{z}$, the wavenumber in the $z$ direction. The solution of the linearized perturbation (of any wavelength) equations takes the so-called normal mode form:

$$
u^{\prime}(x, y, z, t)=\exp \left[\mathrm{i}\left(k_{z} z-\omega t\right)\right] F(x, y) .
$$

The assumption of a constant $k_{z}$ in (2.7) is also consistent with the homogeneity (in $z$ ) of the local stability equations (2.5) and (2.6). To fully establish a correspondence between the solutions in (2.7) for a single-valued function $F(x, y)$ and the solutions of (2.5) and (2.6), one also requires the wavevector $\boldsymbol{k}$ of the WKBJ solution to be periodic when (2.5) is integrated along one period (on the $x y$-plane) of the 3D trajectory. We therefore focus entirely on periodic wavevectors in this paper and, as discussed in $\S \S 3$ and 4 , the wavevector periodicity condition plays a significant role 
in determining the suppression and emergence of instabilities in vortices with an axial flow. The periodicity of $\boldsymbol{k}$ also simplifies the solutions of (2.6) to fall under the Floquet theory for periodic linear differential equations.

\subsection{Periodicity criterion}

To study the stability properties of the base flow, (2.6) is integrated along a given closed streamline in the $x y$-plane for all $\boldsymbol{k}$ that are periodic upon integrating equation (2.5) along that entire streamline once. For each $\boldsymbol{k}$ fulfilling this periodicity condition, the vector amplitude $\boldsymbol{a}$ obeys a Floquet problem once integrated along the streamline over one period. The resulting eigenvalues of the propagator matrix in the Floquet problem give the growth rates and frequencies as a function of the wavevector $\boldsymbol{k}$. As pointed out by Lifschitz \& Hameiri (1991), $\boldsymbol{a} \cdot \boldsymbol{k}$ is conserved upon integrating (2.5) and (2.6) along streamlines.

The integration of (2.5) and (2.6) on a 3D trajectory is parametrized by an integration on its 2D projection on the $x y$-plane, i.e. on closed streamlines of $\psi$. The value of $\psi$ then defines the trajectory chosen, and the time taken by a fluid particle to travel from an initial point to the current point on the trajectory defines the coordinate along the trajectory. To identify all $\boldsymbol{k}$ that are periodic upon integrating equation (2.5) around a specific fluid trajectory, we use the Serret-Frenet decomposition on the projected trajectory in the $x y$-plane:

$$
\boldsymbol{k}=\alpha(t) \boldsymbol{U}_{x y}+\beta(t) \nabla \psi+\gamma(t) \boldsymbol{e}_{z},
$$

where $\alpha(t), \boldsymbol{U}_{x y}, \beta(t), \nabla \psi$ and $\gamma(t)$ are, in general, time-dependent as we integrate equation (2.5) along base flow trajectories.

Along $\boldsymbol{e}_{z},(2.5)$ reduces to:

$$
\mathrm{d} \gamma / \mathrm{d} t=0,
$$

showing that $\gamma$ is constant on the trajectory, as already anticipated from the structure of the global eigenmode in (2.7). For a steady flow, an alternate form of (2.5) is $\mathrm{d}(\boldsymbol{k} \cdot \boldsymbol{U}) / \mathrm{d} t=0$, implying $\boldsymbol{k} \cdot \boldsymbol{U}=\Omega$, where $\Omega$ is a constant. Now,

$$
\boldsymbol{k} \cdot \boldsymbol{U}_{x y}\left(=\alpha|\nabla \psi|^{2}\right)=\boldsymbol{k} \cdot \boldsymbol{U}-\boldsymbol{k} \cdot w \boldsymbol{e}_{z}=\Omega-\gamma w,
$$

resulting in:

$$
\alpha=\frac{\Omega-\gamma w}{|\nabla \psi|^{2}},
$$

implying that $\alpha$ varies since $|\nabla \psi|^{2}$ varies on a trajectory, whereas $\Omega, \gamma$ and $w$ do not. Here $\alpha(t)$, however, is periodic when (2.5) is integrated along a closed streamline.

To derive a criterion imposed by the periodicity of $\beta(t)$, we take the total time derivative of the dot product between (2.8) and $\nabla \psi$ to get:

$$
\begin{aligned}
\frac{\mathrm{d}}{\mathrm{d} t}(\boldsymbol{k} \cdot \nabla \psi) & =\frac{\mathrm{d}\left(\beta|\nabla \psi|^{2}\right)}{\mathrm{d} t}=\beta \frac{\mathrm{d}\left(|\nabla \psi|^{2}\right)}{\mathrm{d} t}+\frac{\mathrm{d} \beta}{\mathrm{d} t}|\nabla \psi|^{2} \\
& =\frac{\mathrm{d} \boldsymbol{k}}{\mathrm{d} t} \cdot \nabla \psi+\boldsymbol{k} \cdot \frac{\mathrm{d} \boldsymbol{\nabla} \psi}{\mathrm{d} t}=-\left[(\nabla \boldsymbol{U})^{\mathrm{T}} \cdot \boldsymbol{k}\right] \cdot \nabla \psi+\boldsymbol{k} \cdot(\boldsymbol{U} \cdot \boldsymbol{\nabla}) \nabla \psi
\end{aligned}
$$


Note that the governing equation (2.5) for $\boldsymbol{k}$ has been used in (2.12). Substituting the expression for $\boldsymbol{k}$ from (2.8), and after some vector algebra, (2.12) reduces to:

$$
\begin{aligned}
\frac{\mathrm{d} \beta}{\mathrm{d} t}= & {\left[-4 \alpha \frac{\partial \psi}{\partial x} \frac{\partial \psi}{\partial y} \frac{\partial^{2} \psi}{\partial x \partial y}-\alpha\left(\left(\frac{\partial \psi}{\partial x}\right)^{2}-\left(\frac{\partial \psi}{\partial y}\right)^{2}\right)\right.} \\
& \left.\times\left(\frac{\partial^{2} \psi}{\partial x^{2}}-\frac{\partial^{2} \psi}{\partial y^{2}}\right)-\gamma \frac{\partial \psi}{\partial x} \frac{\partial w}{\partial x}-\gamma \frac{\partial \psi}{\partial y} \frac{\partial w}{\partial y}\right] /|\nabla \psi|^{2},
\end{aligned}
$$

which when integrated from 0 to $T$ (the period of the streamline we perturb around) should give zero for $\beta$ to be periodic with the same period $T$. Making use of the expression in (2.11), the criterion for the periodicity of $\beta$ can now be stated as:

$$
\alpha I_{1}-\frac{\gamma}{|\nabla \psi|^{2}} I_{2}=0
$$

where

$$
I_{1}=\int_{0}^{T} \frac{-4 \frac{\partial \psi}{\partial x} \frac{\partial \psi}{\partial y} \frac{\partial^{2} \psi}{\partial x \partial y}-\left(\left(\frac{\partial \psi}{\partial x}\right)^{2}-\left(\frac{\partial \psi}{\partial y}\right)^{2}\right)\left(\frac{\partial^{2} \psi}{\partial x^{2}}-\frac{\partial^{2} \psi}{\partial y^{2}}\right)}{|\nabla \psi|^{4}} \mathrm{~d} t
$$

and

$$
I_{2}=\int_{0}^{T} \frac{\frac{\partial \psi}{\partial x} \frac{\partial w}{\partial x}+\frac{\partial \psi}{\partial y} \frac{\partial w}{\partial y}}{|\nabla \psi|^{2}} \mathrm{~d} t=f^{\prime}(\psi) T .
$$

The expressions in (2.1) have been used to analytically evaluate the integral $I_{2}$ in (2.16). The wavevector periodicity criterion in (2.14), a necessary condition to be fulfilled when looking for the WKBJ approximation of an eigenmode, is an alternate form of the periodicity criterion in (4.10) in Lifschitz \& Hameiri (1993). The periodicity criterion in (2.14) simplifies to $\alpha=0$ for any base flow with $\mathrm{d} w / \mathrm{d} \psi=0$ and $I_{1} \neq 0$. Furthermore, for an axisymmetric flow with $\psi(r) \propto r^{2}$ and $\mathrm{d} w / \mathrm{d} r=0$, all wavevectors satisfy the periodicity criterion in (2.14), a scenario considered by Hattori \& Fukumoto (2012).

Since the transformation $\boldsymbol{k}=k_{0} \boldsymbol{k}$ (for any constant $k_{0}$ ) leaves equations (2.5) and (2.6) unchanged, it is sufficient to consider wavevectors of unit magnitude at $t=0$ to identify all the periodic wavevectors that correspond to instabilities (growth of disturbance upon integrating equations (2.5) and (2.6) on a periodic trajectory). We therefore consider a unit initial wavevector of the form

$$
\boldsymbol{k}^{i}=\frac{\cos \theta^{i}}{|\nabla \psi|^{2, i}} \frac{I_{2}}{I_{1}} \boldsymbol{U}_{x y}^{i}+\beta_{ \pm}^{i} \nabla \psi^{i}+\cos \theta^{i} \boldsymbol{e}_{z}
$$

where $\theta^{i}$ is the angle made by the unit vector $\boldsymbol{k}^{i}$ with the $z$-axis (figure 1), and $\beta_{ \pm}^{i}$ is then given by:

$$
\beta_{ \pm}^{i}= \pm \sqrt{\frac{1-\cos ^{2} \theta^{i}}{|\nabla \psi|^{2, i}}-\frac{\cos ^{2} \theta^{i}}{|\nabla \psi|^{4, i}} \frac{I_{2}^{2}}{I_{1}^{2}}}
$$


The superscript $i$ denotes quantities at the initial location $\left(x^{i}, y^{i}\right)$. Note that the periodicity criterion in (2.14) has already been accounted for in (2.17). For the wavevector to be real, $\beta_{ \pm}^{i}$ has to be real, and this condition implies:

$$
\theta^{i} \geqslant \theta_{\text {min }}^{i}=\cos ^{-1} \sqrt{\frac{I_{1}^{2}\left|\nabla \psi^{i}\right|^{2}}{I_{1}^{2}\left|\nabla \psi^{i}\right|^{2}+I_{2}^{2}}},
$$

limiting the range of wavevector angle $\theta^{i}$ for which one may find periodic wavevectors at $\left(x^{i}, y^{i}\right)$ on the chosen trajectory to $\left[\theta_{\min }^{i}, \pi / 2\right]$.

\subsection{Numerical procedure}

Numerically, the trajectory is computed from an initial point $\left(x^{i}, y^{i}\right)$ at $t=0$ by integrating $\mathrm{d} x / \mathrm{d} t=u=-\partial \psi / \partial y$ and $\mathrm{d} y / \mathrm{d} t=v=\partial \psi / \partial x$ using the Runge-Kutta fourth-order scheme with a time step $\Delta t$. To close the trajectory and compute the period $T$, integration is carried out till we reach a time $t=t^{*}$ for which $\mathrm{d}\left(t^{*}\right)=\left(x\left(t^{*}\right)-x^{i}\right)^{2}+\left(y\left(t^{*}\right)-y^{i}\right)^{2}$ attains a local minimum, and the conditions (i) $\left(x\left(t^{*}+\Delta t\right)-x\left(t^{*}\right)\right)\left(x(\Delta t)-x^{i}\right)>0$ and (ii) $\left(y\left(t^{*}+\Delta t\right)-y\left(t^{*}\right)\right)\left(y(\Delta t)-y^{i}\right)>0$ are satisfied. Conditions (i) and (ii) ensure that $t^{*}$ is close to the time period $T$ of the trajectory, and not to some fraction of $T$, where the quantity $\mathrm{d}\left(t^{*}\right)$ can possibly attain a local minimum. The time period $T$ of the periodic trajectory is now more accurately estimated by interpolation as $T=t^{*}+2\left(y^{i}-y\left(t^{*}\right)\right) /\left(v^{i}+v\left(t^{*}\right)\right)$. The periodic trajectory is then re-computed from $\left(x^{i}, y^{i}\right)$ with $\Delta t=T / N$, where $N$ is a large enough integer (chosen to be around 4000 for the results presented in this paper) such that doubling $N$ does not change the magnitude of the growth rates (computed using the procedure described below) up to two decimal places. This step that adjusts $\Delta t$ such that the period $T$ is an integer multiple of $\Delta t$ improves the accuracy of the numerical growth rate calculations.

For each initial position $\left(x^{i}, y^{i}\right)$ chosen on a particular line intersecting all the trajectories (the $x$-axis in the following), growth rate calculations were performed for 1000 different values of the initial angle $\theta^{i}$, distributed equally between $\theta_{\min }^{i}$ and $\pi / 2$. For each $\theta^{i}$, (2.6) is solved (numerically using the Runge-Kutta fourth-order scheme) from $t=0$ to $t=T$ for initial conditions on the amplitude $\boldsymbol{a}_{1}^{i}=\left[\begin{array}{lll}1 & 0 & 0\end{array}\right]$, $\boldsymbol{a}_{\mathbf{2}}^{i}=\left[\begin{array}{lll}0 & 1 & 0\end{array}\right]$ and $\boldsymbol{a}_{\mathbf{3}}^{i}=\left[\begin{array}{lll}0 & 0 & 1\end{array}\right]$ to obtain the amplitude vectors at $t=T$ as $\boldsymbol{a}_{\mathbf{1}}^{f}=\left[\begin{array}{lll}a_{x, 1} & a_{y, 1} & a_{z, 1}\end{array}\right], \boldsymbol{a}_{\mathbf{2}}^{f}=\left[\begin{array}{lll}a_{x, 2} & a_{y, 2} & a_{z, 2}\end{array}\right]$ and $\boldsymbol{a}_{\mathbf{3}}^{f}=\left[\begin{array}{lll}0 & 0 & 1\end{array}\right]$, respectively. As noted in $\$ 2.1$, the amplitudes $\boldsymbol{a}$ aligned with $\boldsymbol{e}_{z}$ correspond to $\mathrm{d} \boldsymbol{a} / \mathrm{d} t=0$, resulting in $\boldsymbol{a}_{\mathbf{3}}^{f}=\boldsymbol{a}_{\mathbf{3}}^{i}$. The growth rate, using results from Floquet theory (Chicone 2000), is then computed as $\sigma=(1 / T) \max \left(\operatorname{Re}\left(\log \left(\lambda_{1,2}\right)\right)\right)$, where $\lambda_{1,2}$ are the eigenvalues of the $2 \times 2$ matrix $\boldsymbol{M}=\left[\begin{array}{llll}a_{x, 1} & a_{x, 2} ; & a_{y, 1} & a_{y, 2}\end{array}\right]$, with the semicolon separating the two rows of the matrix and $\mathrm{Re}$ denoting the real part.

We note here that if $\boldsymbol{a} \cdot \boldsymbol{k}=0(2.4)$ is satisfied at $t=0$ then it remains satisfied for all times when (2.5) and (2.6) are integrated in time. Therefore the plane $\boldsymbol{a} \cdot \boldsymbol{k}^{i}=0$

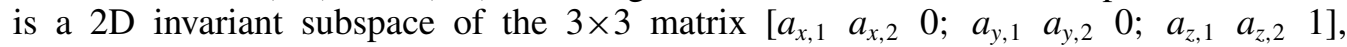
spanned by two eigenvectors satisfying $\boldsymbol{a} \cdot \boldsymbol{k}^{i}=0$. The third eigenvector, corresponding to eigenvalue 1 , is $\boldsymbol{a}_{\mathbf{3}}^{i}=\boldsymbol{a}_{\mathbf{3}}^{f}$. Hence stability is determined by the eigenvalues of the $2 \times 2$ sub-matrix $\left[a_{x, 1} a_{x, 2} ; a_{y, 1} a_{y, 2}\right]$.

\section{Stuart vortices with axial flow}

For the base flow, we consider Stuart vortices (Stuart 1967) with the 2D velocity field $(u, v)$ on the $x y$-plane defined by a $2 \mathrm{D}$ streamfunction:

$$
\psi(x, y)=\log (\cosh y-\rho \cos x),
$$


in the presence of a flow $w(x, y)$ along the axis of the vortex (z-axis). Here, $\rho$ is the concentration parameter and varies between 0 and 1 . Smaller values of $\rho$ correspond to less concentrated vorticity and stronger ellipticity of the streamlines, as depicted in figure 5 of Godeferd et al. (2001). The non-dimensional form of the streamfunction in (3.1) assumes length and velocity scales of $L_{0}$ and $U_{0}$, respectively, to give nondimensional $x, y$ and $\psi$. The corresponding time scale is then given by $T_{0}=L_{0} / U_{0}$.

As discussed in $\S 2$, the steady-flow assumption requires $w$ to be purely a function of $\psi$, i.e. $w(x, y)=f(\psi)$, where $f$ is any smooth function. The spatial derivatives of $w(x, y)$ are then given by:

$$
\begin{aligned}
& \frac{\partial w}{\partial x}=f^{\prime}(\psi) v, \\
& \frac{\partial w}{\partial y}=-f^{\prime}(\psi) u
\end{aligned}
$$

Since the equation for the wavevector $\boldsymbol{k}$ in (2.5), the equation for the amplitude perturbation $\boldsymbol{a}$ in (2.6), and the periodicity criterion for $\boldsymbol{k}$ in (2.14) all depend only on the spatial derivatives of $w(x, y)$, the influence of the axial velocity on the stability of a particular streamline is determined completely by the value of $f^{\prime}(\psi)$. For each streamline, we define a parameter $\tau$ :

$$
\tau=\frac{f^{\prime}(\psi) v\left(x_{0}, 0\right)}{\omega},
$$

where $\omega=(\partial v / \partial x-\partial u / \partial y)$ is the $2 \mathrm{D}$ vorticity on the streamline, i.e. the $z$-component of vorticity, which is invariant on the streamline as a consequence of the Kelvin theorem. Here $x_{0}$ is the point of intersection of the streamline with the positive $x$-axis, allowing us to label the streamline. The parameter $\tau$ is the ratio between the $x$-component of the gradient of axial velocity at a chosen point $\left(x_{0}, 0\right)$ on the streamline and the $z$-component of the vorticity associated with the streamline.

For any axisymmetric flow described by a streamfunction $\psi(r)$, where $r$ is the radial coordinate on the $x y$-plane, the expression for $\tau$ in (3.4) reduces to:

$$
\tau=\left(r \frac{\mathrm{d} w}{\mathrm{~d} r}\right) /\left(\frac{\mathrm{d}}{\mathrm{d} r}\left(r \frac{\mathrm{d} \psi}{\mathrm{d} r}\right)\right) .
$$

For the Stuart vortices, which are non-axisymmetric, $\partial w\left(x_{0}, 0\right) / \partial y=-f^{\prime}(\psi) u\left(x_{0}, 0\right)=0$, and $\tau$ is then the ratio of the axial shear to the $z$-component of vorticity at $\left(x_{0}, 0\right)$. For every $\rho$ and $\tau$, we consider 50 different trajectories, intersecting the $x$-axis at 50 different $\left(x_{0}, 0\right)$, where $x_{0}$ is uniformly distributed between 0 and 3 .

We first examine the dependence of $\theta_{\min }^{i}$ (2.19) on the axial flow, and how, as a consequence, the axial flow reduces the range of acceptable angles $\theta^{i}$ to $\left[\theta_{\min }^{i}, \pi / 2\right]$. In figure 2(a-c), we plot contour lines of $\theta_{\min }^{i}$ on the plane of $x_{0}$ and $\tau$ for $\rho=0.33,0.75$ and 0.9 , respectively. For all $\rho$ and $x_{0}, \theta_{\min }^{i}=0$ for $\tau=0$ and it asymptotically approaches $\pi / 2$ as $\tau$ approaches $\infty$. For the case with strong ellipticity $(\rho=0.33)$, as shown in figure $2(a), \theta_{\min }^{i}$ reaches values close to $\pi / 2$ well before $\tau=1$ for all $x_{0}$. For larger values of $\tau(>1)$ in the $\rho=0.33$ case, there is then a very narrow range of $\theta^{i}\left(\theta_{\min }^{i} \leqslant \theta^{i} \leqslant \pi / 2\right)$ over which one can find periodic wavevectors. For intermediate values of $\rho$, as shown in figure $2(b), \theta_{\min }^{i}$ approaches $\pi / 2$ more slowly for all $x_{0}$ and hence allows for a wider range of periodic wavevector angles even for $\tau>1$. We also note that the convergence to $\theta_{\min }^{i}=\pi / 2$ is the slowest for trajectories 

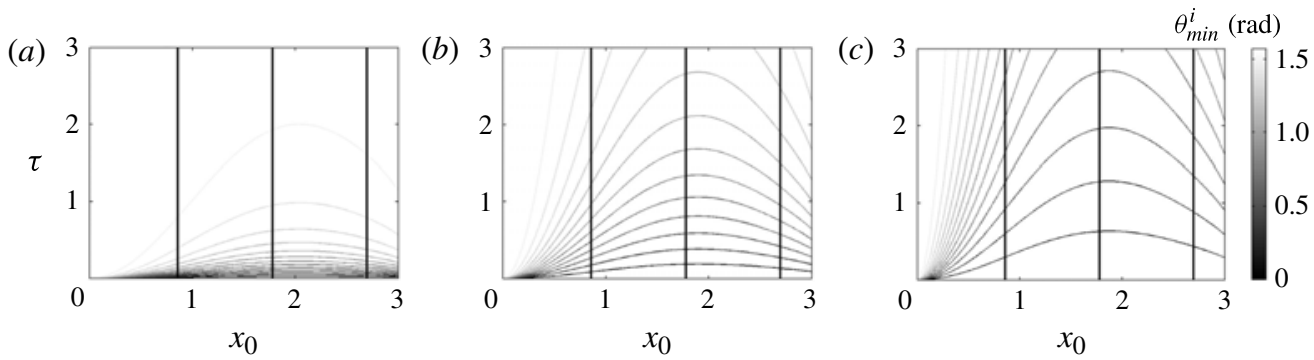

FIGURE 2. Contour lines of $\theta_{\text {min }}^{i}$ on the $x_{0}-\tau$ plane for $(a) \rho=0.33,(b) \rho=0.75$ and (c) $\rho=0.90$. Each plot contains fifteen contour lines, corresponding to values of $\theta_{\min }^{i}$ equispaced between 0 and $\pi / 2$, with $\theta_{\min }^{i}=0$ lying on $\tau=0$. The initial position for all the plots is given by $\left(x^{i}, y^{i}\right)=\left(x_{0}, 0\right)$. The black vertical lines in $(a-c)$ denote $x_{0}=0.85,1.77$ and 2.69, respectively, i.e. the values of $x_{0}$ used in figures 4 and 5 .
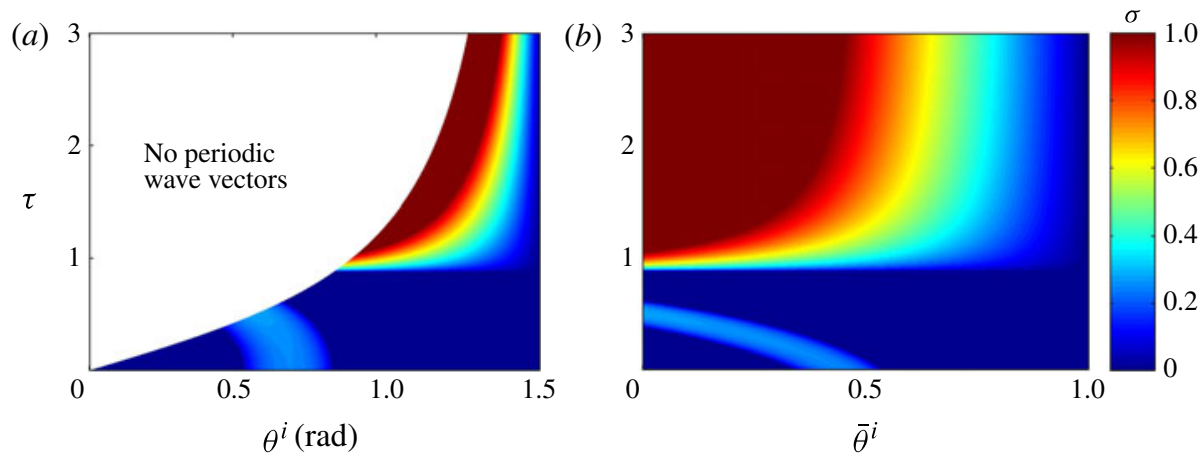

FIGURE 3. (Colour online) (a) Growth rate $\sigma$ as a function of $\theta^{i}$ and $\tau$ for $\rho=0.75$, $x_{0}=0.85$. (b) Growth rate $\sigma$ as a function of $\bar{\theta}^{i}=\left(\theta^{i}-\theta_{\min }^{i}\right) /\left(\pi / 2-\theta_{\min }^{i}\right)$ and $\tau$ for $\rho=0.75, x_{0}=0.85$. The initial position for both the plots is given by $\left(x^{i}, y^{i}\right)=\left(x_{0}, 0\right)$.

around $x_{0} \approx 2$. Finally, the variation of $\theta_{\min }^{i}$ for $\rho=0.9$, as shown in figure $2(c)$, is qualitatively similar to that of $\rho=0.75$, but about three times slower in $\tau$, with faster convergence to $\theta_{\min }^{i}=\pi / 2$ for small $x_{0}$ than for intermediate values of $x_{0}$.

In figure 3(a), we plot the growth rate $\sigma$ as a function of $\theta^{i}$ (which varies between $\theta_{\min }^{i}$ and $\pi / 2$ ) and $\tau$ for $\rho=0.75, x_{0}=0.85$. For $\tau=0$, there is an instability localized around $\theta^{i}=\theta^{*, i}=0.695$, and it has been shown by Godeferd et al. (2001) to correspond to the elliptic instability of the core of the vortex. As $\tau$ increases, $\theta^{*, i}$ (defined as the value of $\theta^{i}$ for which $\sigma$ attains its maximum value $\sigma^{*}$ ) slowly decreases, but the elliptic instability disappears for $\tau \gtrsim \tau_{E}=0.615$ owing to the rapid increase of $\theta_{\text {min }}^{i}$ (see figure $2 b$ ), which defines the boundary of the domain of existence of periodic $\boldsymbol{k}$ solution. In figure $3(b)$, where $\theta^{i}$ has been translated by $\theta_{\min }^{i}$ and rescaled by the bandwidth of possible $\theta^{i}$ to obtain $\bar{\theta}^{i}$, this unstable elliptic branch reaches the boundary $\bar{\theta}^{i}=0$, i.e. $\theta^{i}=\theta_{\text {min }}^{i}$ as $\tau$ is increased from zero and then disappears for $\tau \gtrsim \tau_{E}=0.615$.

In figure 3(a), further increase in $\tau$ beyond $\tau_{E}$ results in the birth of a new branch of instability for $\tau \gtrsim \tau_{C}=0.868$. For this new instability branch, the maximum growth rate occurs for $\theta^{*, i}=\theta_{\text {min }}^{i}$, i.e. $\bar{\theta}^{*, i}=0$, as evidenced in both figure $3(a, b)$. We perform a thorough investigation of this new branch of instability in $\S 4$. 

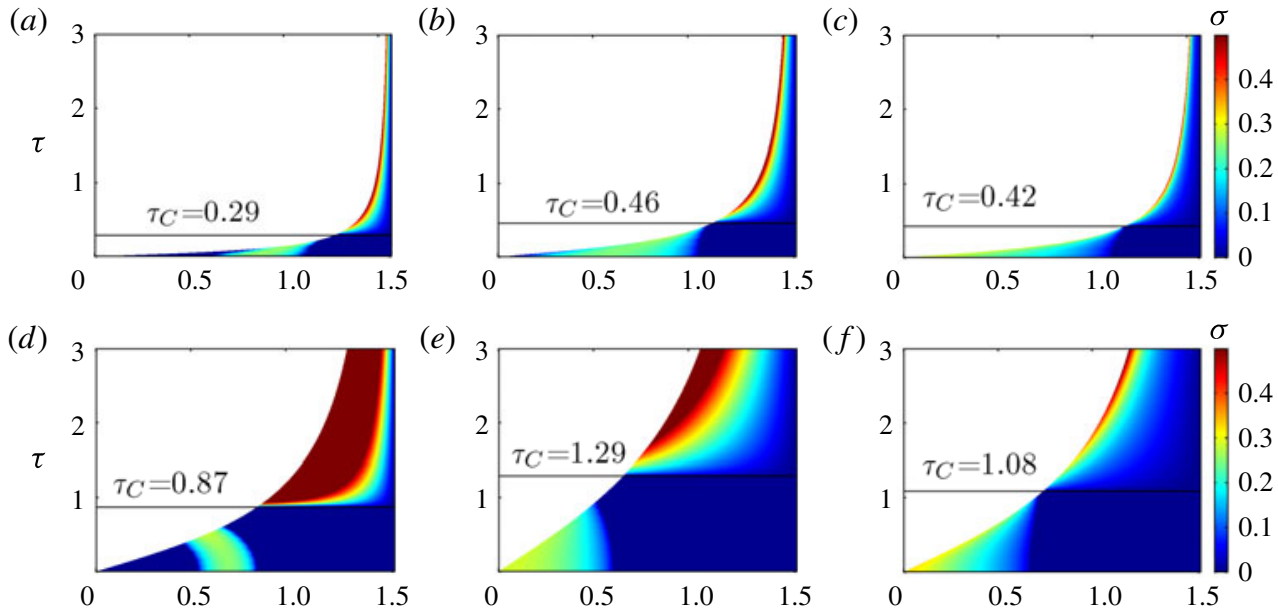

(e)
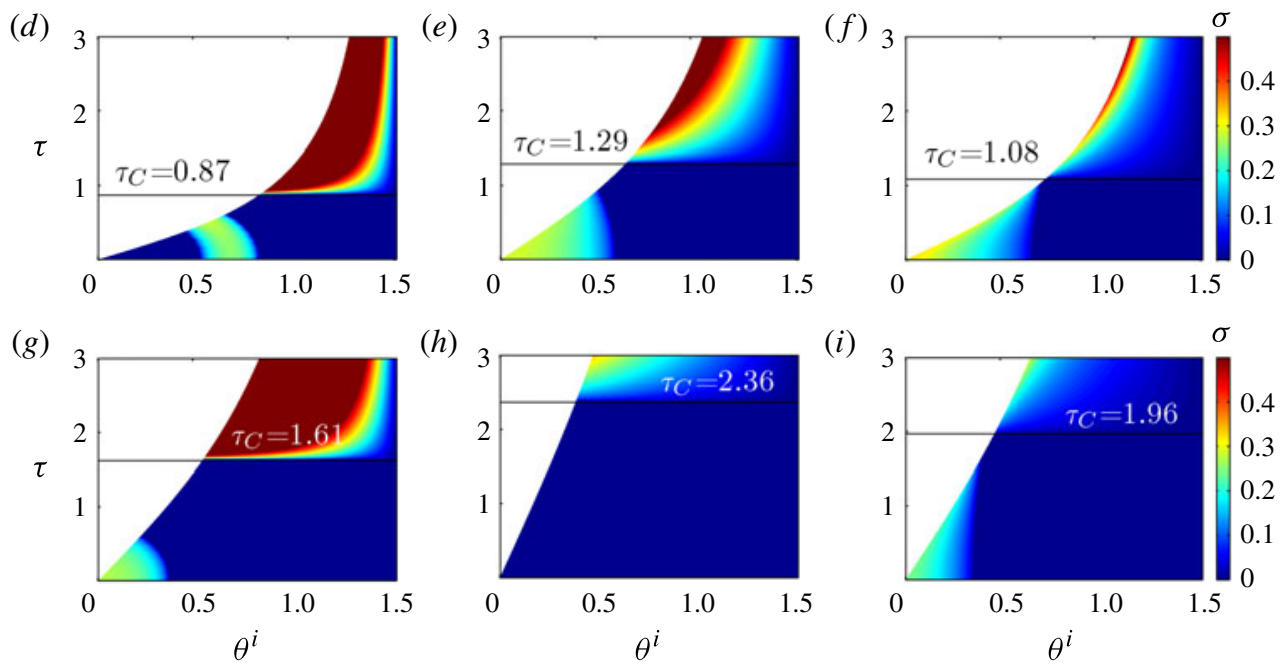

(h)
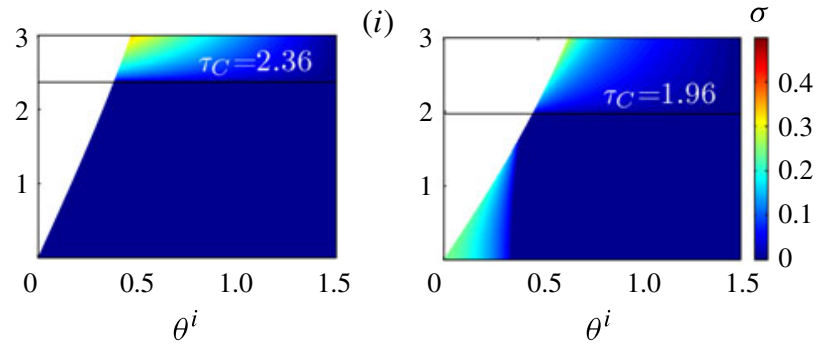

FIgURE 4. (Colour online) Growth rate $\sigma$ as a function of $\theta^{i}$ and $\tau$ for $(a) \rho=0.33$, $x_{0}=0.85 ;(b) \rho=0.33, x_{0}=1.77$; (c) $\rho=0.33, x_{0}=2.69$; (d) $\rho=0.75, x_{0}=0.85$; (e) $\rho=$ $0.75, x_{0}=1.77 ;(f) \rho=0.75, x_{0}=2.69 ;(g) \rho=0.9, x_{0}=0.85 ;(h) \rho=0.9, x_{0}=1.77 ;(i)$ $\rho=0.9, x_{0}=2.69$. The initial conditions for all the plots are given by $\left(x^{i}, y^{i}\right)=\left(x_{0}, 0\right)$.

In figure 4, we plot the growth rate $\sigma$ as a function of $\theta^{i}$ and $\tau$ for the three different values of $\rho$ discussed in figure 2. For each value of $\rho$, results are plotted for three different trajectories, corresponding to three different initial conditions $\left(x^{i}, y^{i}\right)=$ $\left(x_{0}, 0\right)$, indicated by the three black vertical lines in figure 2 . Motivated by the plot in figure $3(b)$, we plot the growth rate $\sigma$ as a function of $\bar{\theta}^{i}=\left(\theta^{i}-\theta_{\min }^{i}\right) /\left(\pi / 2-\theta_{\min }^{i}\right)$ (which varies between 0 and 1) and $\tau$ in figure 5 , for the same set of parameters as in figure 4. For large enough values of $\tau, \theta^{i}$ is restricted to the small range $\theta_{\min }^{i} \leqslant \theta^{i} \leqslant$ $\pi / 2$ owing to $\theta_{\min }^{i}$ being close to $\pi / 2$; the variation of $\sigma$ within this small range of $\theta^{i}$ is more clearly visualized in the plots in figure 5 . The $\tau=0$ sections of all the plots in figures 4 and 5, corresponding to no axial flow, are in complete agreement with the results of Godeferd et al. (2001) for Stuart vortices with no background rotation.

For all three values of $\rho$, the trajectories close to the centre of the vortices $\left(x_{0}=0.85\right.$, figures $4 a$ and $5 a, 4 d$ and $5 d, 4 g$ and $5 g$ ) display the same dynamics and are susceptible to instability for small enough values of $\tau(\tau \gtrsim 0)$. This branch of instability for $\tau=0$, as discussed in figure 3 , corresponds to the elliptic instability and is localized around $\theta^{*, i}=0,0.695$ and 0.844 for $\rho=0.9,0.75$ and 0.33 , respectively. The threshold values of $\tau$, above which this elliptic instability disappears, are $\tau_{E}=0.6,0.615$ and 0.229 for $\rho=0.9,0.75$ and 0.33 , respectively. We note here that $\tau_{E}$ is a function of both $\rho$ and $x_{0}$ in the domain of $x_{0}$ where elliptic instability is present without an axial flow according to Godeferd et al. (2001). 

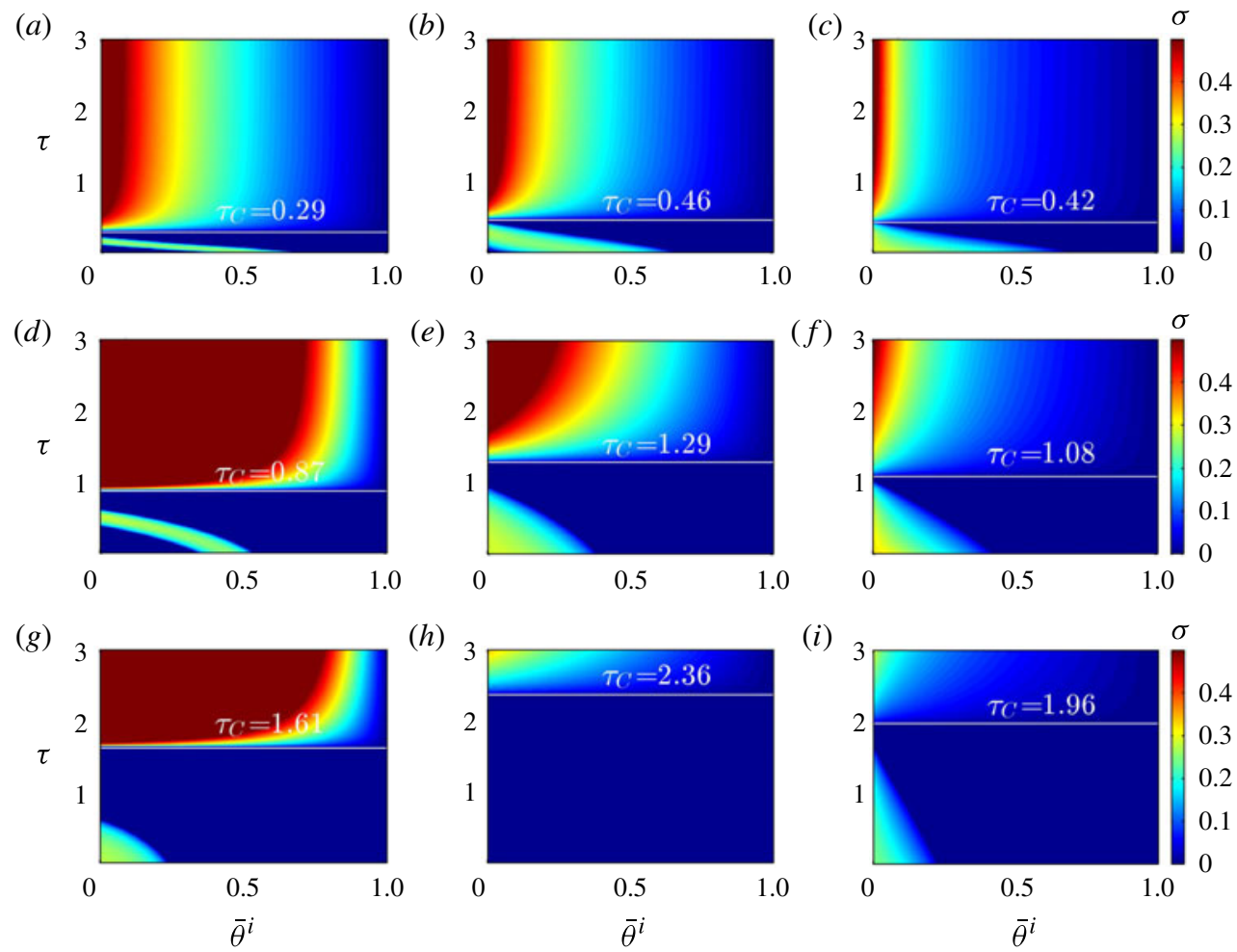

(h)
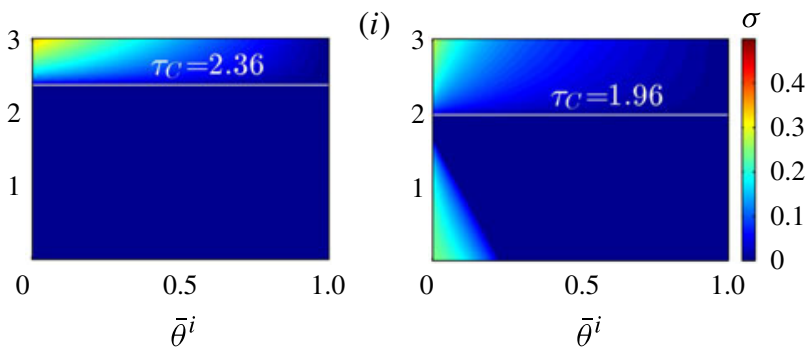

FIGURE 5. (Colour online) Growth rate $\sigma$ as a function of $\bar{\theta}^{i}=\left(\theta^{i}-\theta_{\text {min }}^{i}\right) /\left(\pi / 2-\theta_{\text {min }}^{i}\right)$ and $\tau$ for $(a) \rho=0.33, x_{0}=0.85$; (b) $\rho=0.33, x_{0}=1.77$; (c) $\rho=0.33, x_{0}=2.69$; (d) $\rho=$ $0.75, x_{0}=0.85 ;$ (e) $\rho=0.75, x_{0}=1.77 ;(f) \rho=0.75, x_{0}=2.69 ;(g) \rho=0.90, x_{0}=0.85$; (h) $\rho=0.90, x_{0}=1.77$; (i) $\rho=0.90, x_{0}=2.69$. The initial conditions for all the plots are given by $\left(x^{i}, y^{i}\right)=\left(x_{0}, 0\right)$.

Further increase in $\tau$, as discussed earlier for $\rho=0.75, x_{0}=0.85$ in figure 3 , results in the appearance of a new branch of instability with $\theta^{*, i}=\theta_{\min }^{i}$, i.e. $\bar{\theta}^{*, i}=0$ for $\tau \geqslant \tau_{C}$. This new branch appears for all values of the vortex concentration parameter $\rho$ and streamlines labeled by $x_{0}$, as shown in figures $4(a-i)$ and $5(a-i)$. The threshold $\tau_{C}$, defined for all $\rho$ and $x_{0}$, increases with $\rho$ but varies less with $x_{0}$, with a slight increase between $x_{0}=0.85$ and $x_{0}=1.77$, and then a decrease for $x_{0}=2.69$. Table 1 summarises the values of $\tau_{C}$ for all the cases shown in figures 4 and 5 .

For all three values of $\rho$, the trajectories far from the centre of the vortices $\left(x_{0}=\right.$ 2.69, figures $4 c$ and $5 c, 4 f$ and $5 f, 4 i$ and $5 i$ ) and close to the hyperbolic point at $x_{0}=\pi$ are susceptible to the hyperbolic instability for $\tau=0$, as discussed in Godeferd et al. (2001). This branch of hyperbolic instability is then characterized by $\theta^{*, i}=\theta_{\min }^{i}$, i.e. $\bar{\theta}^{*, i}=0$ when $\tau$ is small enough, with the maximum growth rate occurring at $\theta^{i}=\theta^{*, i}=0$ for $\tau=0$. The maximum growth rate of this hyperbolic instability branch is strongly affected by an increase in $\tau$ as $\theta_{\min }^{i}$, as shown in figure 2, increases with $\tau$.

In the large core size case $(\rho=0.33)$, as shown in figure 5(c), the range of unstable $\left(\theta^{i}-\theta_{\min }^{i}\right) /\left(\pi / 2-\theta_{\min }^{i}\right)$ corresponding to the hyperbolic instability branch decreases as $\tau$ is increased from 0 , before getting completely suppressed beyond a threshold value of $\tau=\tau_{H}$. Further increase in $\tau$ results in the appearance of the new branch of instability for $\tau \geqslant \tau_{C}$, as discussed earlier. For the cases with more concentrated vortex 


\begin{tabular}{cccc} 
& \multicolumn{3}{c}{$x_{0}$} \\
\cline { 2 - 4 }$\rho$ & 0.85 & 1.77 & 2.69 \\
0.33 & 0.29 & 0.46 & 0.42 \\
0.75 & 0.87 & 1.29 & 1.08 \\
0.90 & 1.61 & 2.36 & 1.96
\end{tabular}

TABLE 1. Axial shear threshold $\tau_{C}$ for the occurrence of the new instability.

cores ( $\rho=0.75$ in figures $4 f$ and $5 f$ and $\rho=0.9$ in figures $4 i$ and $5 i)$, we observe features qualitatively similar to those of $\rho=0.33$, with the hyperbolic branch being suppressed for $\tau>\tau_{H}, \tau_{H}$ being a monotonically increasing function of $\rho$.

Intermediate trajectories that are neither too close nor too far from the centre are subject to a combination of the elliptic and hyperbolic instabilities for $\tau=0$ (Godeferd et al. 2001) that carry over for small $\tau$ till they are suppressed (primarily by an increase in $\left.\theta_{\text {min }}^{i}\right)$ as $\tau$ increases further. For the case of $\left(\rho, x_{0}\right)=(0.33,1.77)$ shown in figures $4(b)$ and $5(b)$, the elliptic instability dominates since $\theta^{*, i}>0$ for $\tau=0$, similar to the case of $\left(\rho, x_{0}\right)=(0.33,0.85)$ shown in figures $4(a)$ and $5(a)$. Strictly speaking, pure elliptic instability for $\tau=0$ can only be observed for $x_{0}=0$ with $\theta^{*, i} \approx \pi / 3$; the value of $\theta^{*, i}$ shifts from $\pi / 3$ as $x_{0}$ increases from zero and, by continuity in $x_{0}$, we refer to this branch as the elliptic branch. This elliptic instability branch for $\tau=0$ may sometimes correspond to $\theta^{*, i}=0$ for trajectories sufficiently far from the centre, one such example being shown in figures $4(\mathrm{~g})$ and $5(\mathrm{~g})$.

For the case of $\left(\rho, x_{0}\right)=(0.75,1.77)$ shown in figures $4(e)$ and $5(e)$, the hyperbolic instability dominates since $\theta^{*, i}=0$ for $\tau=0$. The hyperbolic instability, characterized by a maximum growth rate at $\theta^{*, i}=0$, starts at the hyperbolic point $x_{0}=\pi$ and continues over to smaller $x_{0}$, with the maximum growth rate occurring at $\theta^{*, i}=0$. The reader is referred to Godeferd et al. (2001) for a thorough discussion.

For $\rho=0.9$, the intermediate trajectory $x_{0}=1.77$ is outside the vortex core and away from the hyperbolic point. As shown by Godeferd et al. (2001) this trajectory is stable for all angles $\theta^{i}$, as confirmed by the deep blue colour on the axis $\tau=0$ in figures $4(h)$ and $5(h)$. When the axial velocity shear $\tau$ is increased, this streamline continues being stable up to $\tau<\tau_{C}$, where $\tau_{C}$ is the threshold value of $\tau$ above which the new branch of instability with $\theta^{*, i}=\theta_{\min }^{i}$ appears (white horizontal line in figure $5 h$ ).

In summary, for all three values of $x_{0}$ and $\rho$, instabilities that exist with no axial flow $(\tau=0)$ persist till a threshold value of $\tau\left(=\tau_{E}\right.$ or $\tau_{H}$ depending on the nature of the instability). Specifically, we observe the suppression of the elliptic instability in figures $4(a)$ and $5(a), 4(b)$ and $5(b), 4(d)$ and $5(d)$ and $4(g)$ and $5(g)$, and a suppression of the hyperbolic instability in figures $4(c)$ and $5(c), 4(e)$ and $5(e), 4(f)$ and $5(f)$ and $4(i)$ and $5(i)$. Further increase in $\tau$ results in the appearance of a new branch of instability at $\tau=\tau_{C}$, which, for all three values of $x_{0}$, increases monotonically with $\rho$. The axial velocity shear has, for small values, a stabilizing effect on both the elliptic and hyperbolic instabilities that exist with no axial flow and then, at larger values, a destabilizing effect with the maximum growth rate occurring at the lower limit $\theta_{\min }^{i}$ of the allowed range of the wavevector angle. Our results are qualitatively consistent with those of Hattori \& Hijiya (2010) for the Hill's vortex with an axial flow.

The leading instability, defined as the one that corresponds to the maximum growth rate for fixed values of $\rho, x_{0}$ and $\tau$, is now systematically studied for the same three 

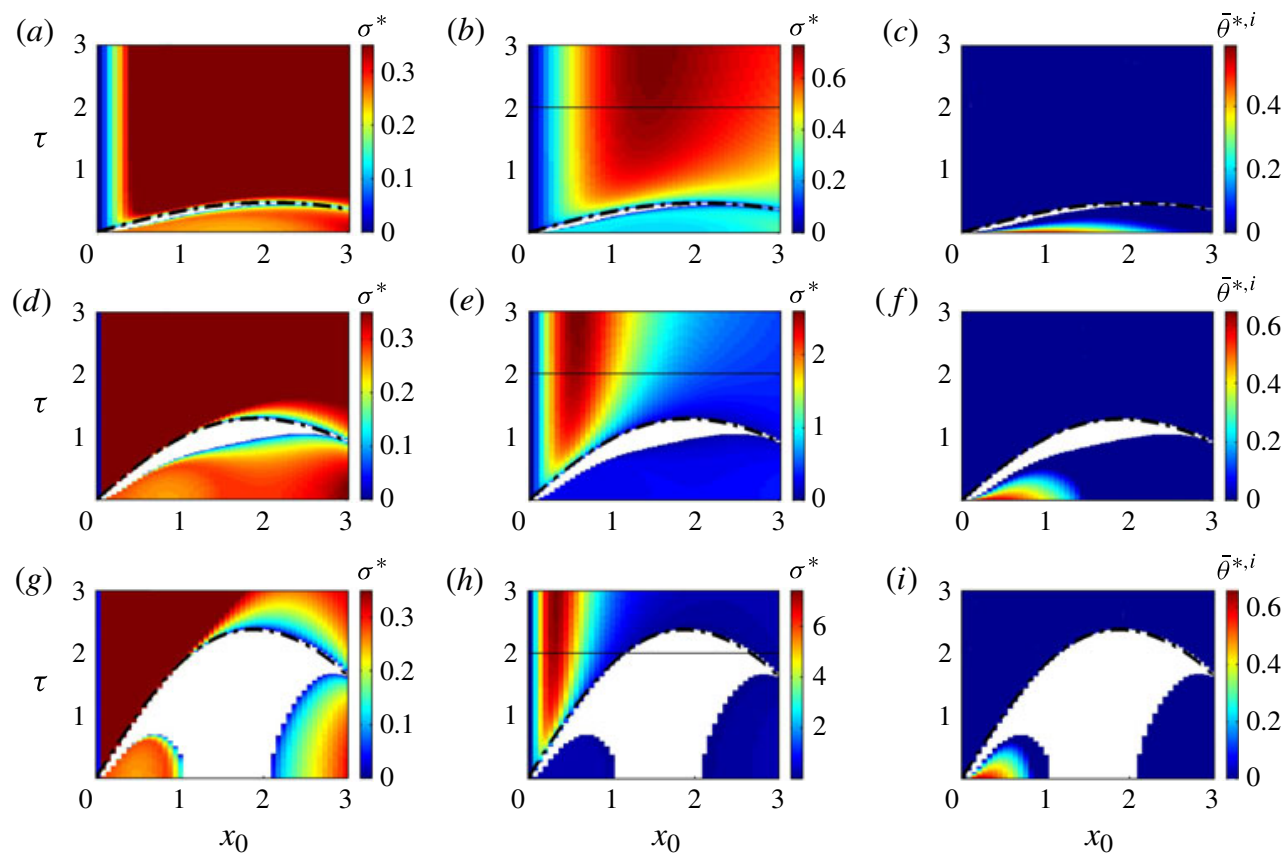

FIgURE 6. (Colour online) $(a, b, d, e, g, h)$ Maximum growth rate $\sigma^{*}$ as a function of $x_{0}$ and $\tau$ for $(a, b) \rho=0.33$, $(d, e) \rho=0.75$ and $(g, h) \rho=0.9$. Figures in the first and second columns differ only in the scale of the colour bar, thus bringing out all the features present. $(c, f, i) \bar{\theta}^{*, i}=\left(\theta^{*, i}-\theta_{\text {min }}^{i}\right) /\left(\pi / 2-\theta_{\text {min }}^{i}\right)$ as a function of $x_{0}$ and $\tau$ for $(c) \rho=0.33,(f) \rho=$ 0.75 and $(i) \rho=0.9$. The initial conditions for all the plots are given by $\left(x^{i}, y^{i}\right)=\left(x_{0}, 0\right)$. The dashed black curves denote the threshold $\tau=\tau_{C}$, above which the heuristic criterion equation (4.16) predicts centrifugal instability. The solid black horizontal lines denote $\tau=2$, the sections along which $\sigma^{*}$ is plotted in figure 7 .

values of the vortex concentration parameter $\rho$ for all the streamlines indexed by $x_{0}$. In the first two columns of figure 6, we plot the maximum growth rate $\sigma^{*}$ (the maximum of $\sigma$ over all the allowable values of the wavevector angle, $\theta_{\min }^{i} \leqslant \theta^{i} \leqslant \pi / 2$ ) as a function of $x_{0}$ and $\tau$. The second column replicates the first but with a different scale of the colour bar to bring out the various features, since, as discussed below, different instabilities have different scalings.

The last column of figure 6 shows the wavevector angle $\theta^{*, i}$ that corresponds to the maximum growth rate. As was done for the plots in figure $5, \theta^{*, i}$ is translated by $\theta_{\min }^{i}$ and rescaled by $\pi / 2-\theta_{\min }^{i}$ to obtain $\bar{\theta}^{*, i}=\left(\theta^{*, i}-\theta_{\min }^{i}\right) /\left(\pi / 2-\theta_{\min }^{i}\right)$ in order to make visible the region where $\theta_{\min }^{i}$ asymptotes to $\pi / 2$. As shown in figure 2 , we recall that $\theta_{\min }^{i}=0$ for $\tau=0$ in the absence of axial flow and $\theta_{\min }^{i}$ tends to $\pi / 2$ for large $\tau$; convergence to $\theta_{\text {min }}^{i}=\pi / 2$ is faster for weakly concentrated vortices $(\rho=0.33)$ than for strongly concentrated vortices $(\rho=0.9)$, for which even at $\tau=3, \theta_{\text {min }}^{i}$ is smaller than one for $x_{0}>0.7$. The white region in all the plots of figure 6 is the stable region.

For the strongly concentrated vortex with $\rho=0.9$, in the absence of axial flow, i.e. $\tau=0$ (figure $6 g$ ) the instability is split between two domains: inside the vortex core for $x_{0} \lesssim 1$ and close to the hyperbolic point $2.1 \lesssim x_{0} \leqslant \pi$, respectively associated with elliptic and hyperbolic instability since $\theta^{*, i}$ is non-zero for the small $x_{0}$ domain and zero for $x_{0}$ close to $\pi$ (figure $6 i$ ). For the trajectories $0.8 \lesssim x_{0} \lesssim 1$, the maximum 
growth rate occurs for $\theta^{*, i}=0$, but the corresponding instability is still categorized as elliptic as it is a continuation of the elliptic branch that exists for smaller $x_{0}$.

When $\tau$ is increased from zero, these two instabilities continue to exist but shrink to a smaller range of $x_{0}$, with the elliptic instability of the core of the vortex stabilized first. With a further increase in $\tau$, a new branch of unstable mode appears in the core of the vortices starting at $x_{0}=0$ with $\theta^{*, i}=\theta_{\text {min }}^{i}$ (figure $6 i$ ) and $\sigma^{*}$ increasing extremely rapidly (saturated colour in figure $6 g$ ), made visible by a change in the scale of the colour bar in figure $6(h)$, where the same data as in figure $6(g)$ is plotted.

For a less concentrated core of the vortices $\rho=0.75$ (figure $6 d-f$ ) and $\rho=0.33$ (figure $6 a-c$ ) the same features are visible except that, in the absence of axial flow $(\tau=0)$, all the $x_{0}$ are unstable and the two domains of instability, mainly associated with the elliptic instability in the core of the vortices (small $x_{0}$ ) and to the hyperbolic instability for $x_{0}$ close to $\pi$, are now connected. Increasing the axial velocity shear $\tau$ results in the stabilization of this joined domain, starting near the core of the vortices ( $x_{0}$ close to zero) first. The new unstable branch with $\theta^{*, i}=\theta_{\min }^{i}$ (figure $6 c, f$ ) appears in the core of the vortices and extends to the entire domain more rapidly (i.e. for smaller $\tau$ ) when the vortices are less concentrated. All the closed streamlines (i.e. all the $x_{0}$ between 0 and $\pi$ ) are unstable above $\tau_{C}^{*}=0.48,1.3$ and 2.38 for $\rho=0.33,0.75$ and 0.90 , respectively.

In all the $\sigma^{*}$ plots, for a given $x_{0}$, one can always identify a threshold of $\tau$ above which a new branch of instability, with growth rates typically larger than the elliptic and hyperbolic instabilities, is born. This new branch of instability always corresponds to $\theta^{*, i}=\theta_{\min }^{i}$. In the next section, we show that this is associated with the centrifugal instability branch.

\section{Discussion}

Based on the observation that $\theta^{*, i}=\theta_{\text {min }}^{i}$ for the new branch of instability for all three values of $\rho$, we now investigate the conjecture that the new instability appearing for large enough $\tau\left(\tau>\tau_{C}\right)$ is a centrifugal instability. To do so, we first consider an axisymmetric vortex with an axial flow and calculate the values for $\theta_{\min }^{i}$ and $\sigma$, with $\sigma^{*}$ to be compared with the predictions of the centrifugal instability theory by Leibovich \& Stewartson (1983). In order to isolate the effects of an axial velocity on the centrifugal instability, we first study axisymmetric base flows as they are not susceptible to elliptic and hyperbolic instabilities.

\subsection{Axisymmetric flows}

For an axisymmetric base flow specified by the streamfunction $\psi(r)$ and axial velocity $w(r)=f(\psi), \partial \psi / \partial x=\dot{\psi} \cos \phi, \partial \psi / \partial y=\dot{\psi} \sin \phi, \partial^{2} \psi / \partial x^{2}=\cos ^{2} \phi(\ddot{\psi}-\dot{\psi} / r)+\dot{\psi} / r$, $\partial^{2} \psi / \partial y^{2}=\sin ^{2} \phi(\ddot{\psi}-\dot{\psi} / r)+\dot{\psi} / r$ and $\partial^{2} \psi / \partial x \partial y=\sin \phi \cos \phi(\ddot{\psi}-\dot{\psi} / r)$, where $r$ and $\phi$ are the radial and azimuthal coordinates, respectively and the upper dot in $\dot{a}$ denotes derivative of any function $a$ with respect to $r$. Recognizing that $\mathrm{d} t=r \mathrm{~d} \phi / \dot{\psi}$ for integration around a circular trajectory of radius $r, \dot{\psi}$ being the azimuthal velocity, the integrals in (2.15) and (2.16) defining the lower limit $\theta_{\min }^{i}$ of the wavevector to be periodic (2.19) reduce to:

$$
I_{1}=\frac{-2 \pi r}{\dot{\psi}^{3}}\left(\ddot{\psi}-\frac{\dot{\psi}}{r}\right)
$$

and

$$
I_{2}=f^{\prime} T=\frac{2 \pi r \dot{w}}{\dot{\psi}^{2}},
$$


where $f^{\prime}=\mathrm{d} f / \mathrm{d} \psi$ as defined previously. Substituting the expressions in (4.1) and (4.2) in (2.14), we get:

$$
\alpha=\frac{-\gamma \dot{w}}{\dot{\psi}(\ddot{\psi}-\dot{\psi} / r)},
$$

which is the same as (5.6) in Leibovich \& Stewartson (1983) with their axial wavenumber $\alpha=\gamma$ and their azimuthal wavenumber $n=r \alpha \psi^{\prime}$. We find it intriguing that the criterion for stationary ' $\gamma$ ' (and maximum growth rate) in Leibovich \& Stewartson (1983) and our criterion for periodic wavevectors match. The minimum angle $\theta_{\min }^{i}$ (2.19) above which periodic wavevectors exist is given by:

$$
\theta_{\text {min }}^{i}=\cos ^{-1} \sqrt{\frac{(r \ddot{\psi}-\dot{\psi})^{2}}{(r \ddot{\psi}-\dot{\psi})^{2}+r^{2} \dot{w}^{2}}} .
$$

To solve (2.6), we evaluate its right hand side in cylindrical coordinates:

$$
\begin{aligned}
& -\boldsymbol{\nabla} \boldsymbol{U} \cdot \boldsymbol{a}+\frac{2}{|\boldsymbol{k}|^{2}}[(\boldsymbol{U} \cdot \boldsymbol{a}) \cdot \boldsymbol{k}] \boldsymbol{k} \\
& \quad=\left[\begin{array}{ccc}
2 \beta \dot{\psi}(\alpha \dot{\psi} \ddot{\psi}+\gamma \dot{w}) & \dot{\psi} / r-2 \beta^{2} \dot{\psi}^{3} / r & 0 \\
-\ddot{\psi}+2 \alpha \dot{\psi}(\alpha \dot{\psi} \ddot{\psi}+\gamma \dot{w}) & -2 \alpha \beta \dot{\psi}^{3} / r & 0 \\
-\dot{w}+2 \gamma(\alpha \dot{\psi} \ddot{\psi}+\gamma \dot{w}) & -2 \gamma \beta \dot{\psi}^{2} / r & 0
\end{array}\right]\left[\begin{array}{l}
a_{r} \\
a_{\theta} \\
a_{z}
\end{array}\right],
\end{aligned}
$$

where the amplitude vector has been written as $\boldsymbol{a}=a_{r} \boldsymbol{e}_{\boldsymbol{r}}+a_{\theta} \boldsymbol{e}_{\theta}+a_{z} \boldsymbol{e}_{z}$, and $\alpha, \beta$ and $\gamma$ are as defined in (2.8) with

$$
\dot{\psi}^{2}\left(\left(\alpha^{i}\right)^{2}+\left(\beta^{i}\right)^{2}\right)+\left(\gamma^{i}\right)^{2}=1
$$

for initial wavevectors of unit magnitude. Now, recognizing that $\mathrm{d} \boldsymbol{e}_{\boldsymbol{r}} / \mathrm{d} t=(\dot{\psi} / r) \boldsymbol{e}_{\theta}$ and $\mathrm{d} \boldsymbol{e}_{\theta} / \mathrm{d} t=(-\dot{\psi} / r) \boldsymbol{e}_{\boldsymbol{r}}$, (2.6) reduces, after making use of the periodicity condition in (4.3), to:

$$
\left[\begin{array}{l}
\mathrm{d} a_{r} / \mathrm{d} t \\
\mathrm{~d} a_{\theta} / \mathrm{d} t \\
\mathrm{~d} a_{z} / \mathrm{d} t
\end{array}\right]=\left[\begin{array}{ccc}
2 \alpha \beta \dot{\psi}^{3} / r & 2 \dot{\psi} / r-2 \beta^{2} \dot{\psi}^{3} / r & 0 \\
-\ddot{\psi}+2 \alpha^{2} \dot{\psi}^{3} / r-\dot{\psi} / r & -2 \alpha \beta \dot{\psi}^{3} / r & 0 \\
-\dot{w}+2 \gamma \alpha \dot{\psi}^{2} / r & -2 \gamma \beta \dot{\psi}^{2} / r & 0
\end{array}\right]\left[\begin{array}{c}
a_{r} \\
a_{\theta} \\
a_{z}
\end{array}\right]
$$

which in vector form can be written as $\mathrm{d} \boldsymbol{a} / \mathrm{d} t=\boldsymbol{C} \boldsymbol{a}$, where $\boldsymbol{C}$ is the coefficient matrix in (4.7). Since $\alpha, \beta$ and $\gamma$ are invariant along a fluid trajectory for periodic wavevectors in axisymmetric flows, the eigenvalues of $\boldsymbol{C}$ represent the growth rates. One of the three eigenvalues of $\boldsymbol{C}$ is $\lambda_{1}=0$, while the remaining two eigenvalues $\lambda_{2,3}$ are the solutions of:

$$
\lambda^{2}=\frac{-2 \dot{\psi}}{r}\left(\ddot{\psi}+\frac{\dot{\psi}}{r}\right)+\frac{4 \dot{\psi}^{4}}{r^{2}} \alpha^{2}+\frac{2 \beta^{2} \dot{\psi}^{3}}{r}\left(\ddot{\psi}+\frac{\dot{\psi}}{r}\right) .
$$

Conditions in (4.3) and (4.6) give:

$$
\alpha^{2}=\frac{r^{2} \dot{w}^{2}}{(r \ddot{\psi}-\dot{\psi})^{2}+r^{2} \dot{w}^{2}}\left(\frac{1}{\dot{\psi}^{2}}-\beta^{2}\right),
$$


which on substitution in (4.8) reduces it to:

$$
\lambda^{2}=-2 r^{2} \dot{\psi} \frac{\mathrm{d}}{\mathrm{d} r}\left(\frac{\dot{\psi}}{r}\right) \frac{\dot{w}^{2}+\frac{\mathrm{d}}{\mathrm{d} r}(r \dot{\psi}) \frac{\mathrm{d}}{\mathrm{d} r}\left(\frac{\dot{\psi}}{r}\right)}{(r \ddot{\psi}-\dot{\psi})^{2}+r^{2} \dot{w}^{2}}\left(1-\beta^{2} \dot{\psi}^{2}\right) .
$$

Since $\beta^{2} \dot{\psi}^{2} \leqslant 1$ as a result of (4.6), $\lambda^{2}>0$ requires:

$$
\dot{\psi} \frac{\mathrm{d}}{\mathrm{d} r}\left(\frac{\dot{\psi}}{r}\right) \frac{\dot{w}^{2}+\frac{\mathrm{d}}{\mathrm{d} r}(r \dot{\psi}) \frac{\mathrm{d}}{\mathrm{d} r}\left(\frac{\dot{\psi}}{r}\right)}{(r \ddot{\psi}-\dot{\psi})^{2}+r^{2} \dot{w}^{2}}<0,
$$

specifying the necessary and sufficient condition for short-wavelength instability in an axisymmetric flow. The growth rate $\lambda_{\max }$ attains a maximum for $\beta=0$, which in turn corresponds to:

$$
\gamma^{2}=\frac{(r \ddot{\psi}-\dot{\psi})^{2}}{(r \ddot{\psi}-\dot{\psi})^{2}+r^{2} \dot{w}^{2}},
$$

specifying the angle between the most unstable wavevector and the $z$-axis. We note that the most unstable wavevector was not explicitly discussed and shown in the papers by Eckhoff \& Storesletten (1978) and Leblanc \& Le Duc (2005).

The criterion in (4.11) for a circular trajectory in an axisymmetric flow to be unstable to short-wavelength perturbations coincides with the sufficient condition for instability derived by Leibovich \& Stewartson (1983). The corresponding maximum growth rate $\sigma^{*}$ is reached for $\beta=0$ in (4.10):

$$
\sigma^{* 2}=-2 r^{2} \dot{\psi} \frac{\mathrm{d}(\dot{\psi} / r)}{\mathrm{d} r} \frac{\dot{w}^{2}+(\mathrm{d} / \mathrm{d} r)(r \dot{\psi})(\mathrm{d} / \mathrm{d} r)(\dot{\psi} / r)}{(r \ddot{\psi}-\dot{\psi})^{2}+r^{2} \dot{w}^{2}},
$$

which coincides with the maximum growth rate expression equation (5.8) in Leibovich \& Stewartson (1983) for particular perturbations with constant value of the frequency of the perturbation in the frame moving with the fluid (5.6), giving:

$$
\sigma^{* 2}=2 v_{\theta} \frac{\left(r \dot{v}_{\theta}-v_{\theta}\right)\left(v_{\theta}^{2} / r^{2}-\dot{v}_{\theta}^{2}-\dot{w}^{2}\right)}{\left(r \dot{v}_{\theta}-v_{\theta}\right)^{2}+r^{2} \dot{w}^{2}} .
$$

To generalize the centrifugal instability criterion in (4.11) to non-axisymmetric flows, we rewrite the criterion as:

$$
\frac{\mathrm{d}}{\mathrm{d} \psi}(\dot{\psi} / r)\left(\left(\frac{\mathrm{d} w}{\mathrm{~d} \psi}\right)^{2}+\frac{\mathrm{d}(r \dot{\psi})}{\mathrm{d} \psi} \frac{\mathrm{d}(\dot{\psi} / r)}{\mathrm{d} \psi}\right)<0,
$$

where $\mathrm{d} / \mathrm{d} r$ in (4.11) has been replaced by $\dot{\psi} \mathrm{d} / \mathrm{d} \psi$. We now choose to replace $\dot{\psi} / r$ by $2 \pi / T$ and $r \dot{\psi}$ by $\Gamma / 2 \pi$, where $T$ and $\Gamma$ are the time period and circulation of the closed fluid trajectory, respectively. These replacements are motivated by (i) the significant roles of $\Gamma$ and $\psi$ in the stability of 2C2D base flows (Bayly 1988), and (ii) the time period $T$ being a crucial factor in the wavevector periodicity criterion (2.16). The centrifugal instability criterion now reduces to:

$$
\frac{\mathrm{d} T}{\mathrm{~d} \psi}\left(\left(\frac{\mathrm{d} w}{\mathrm{~d} \psi}\right)^{2}-\frac{1}{T^{2}} \frac{\mathrm{d} \Gamma}{\mathrm{d} \psi} \frac{\mathrm{d} T}{\mathrm{~d} \psi}\right)>0
$$



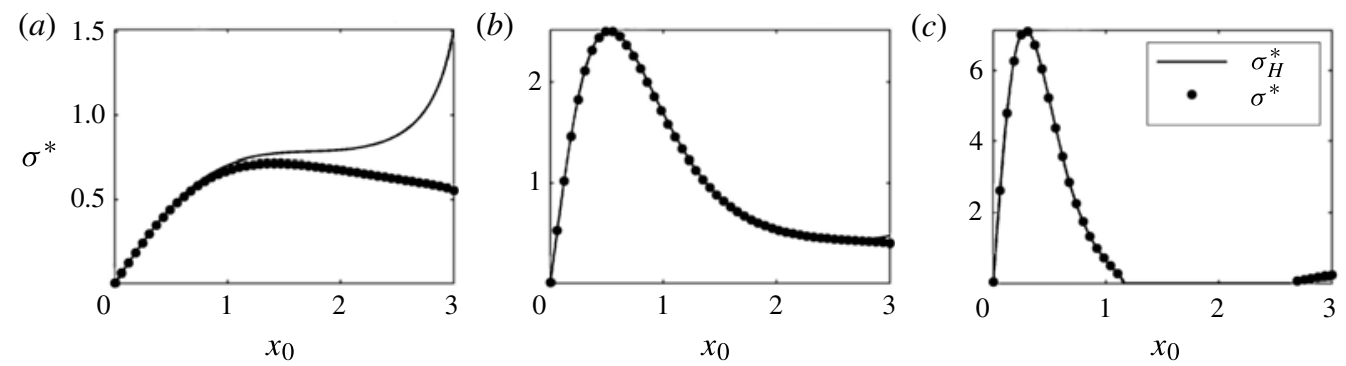

FIGURE 7. Maximum growth rate $\sigma^{*}$ as a function of $x_{0}$ for $(a) \rho=0.33$, (b) $\rho=0.75$ and (c) $\rho=0.9$. All plots correspond to $\tau=2$, the horizontal sections indicated by the black lines in figure 6 . Here $\sigma_{H}^{*}$ is the maximum growth rate predicted by the heuristic criterion in (4.17).

an expression that can be evaluated for non-axisymmetric flows, with $\Gamma$ being defined as: $\Gamma=\oint_{C} \boldsymbol{U}_{\boldsymbol{B}} \cdot \boldsymbol{d} \boldsymbol{l}$, where $\boldsymbol{d} \boldsymbol{l}$ is the vector representing a differential length along the streamline. For trajectories that wind around in the clockwise direction on the $x y$-plane, $T$ and $\Gamma$ are both negative. The above heuristic criterion for centrifugal instability also suggests that an alternate non-dimensional measure (instead of $\tau$ ) of the axial flow is $T^{2}(\mathrm{~d} w / \mathrm{d} \psi)^{2}(\mathrm{~d} \Gamma / \mathrm{d} \psi)^{-1}(\mathrm{~d} T / \mathrm{d} \psi)^{-1}$. We further note that the criterion in (4.16), for flows with $\mathrm{d} w / \mathrm{d} \psi=0$, reduces to $\mathrm{d} \Gamma / \mathrm{d} \psi<0$, i.e. the magnitude of the circulation decreases outwards for a convex closed streamline, a result derived by Bayly (1988). The criterion in (4.16) is invariant with the choice of $L_{0}$ and $U_{0}$, the length and velocity scales used to non-dimensionalize the base flow.

To evaluate the validity of (4.16) for non-axisymmetric flows, in figure 6, we plot (dashed curves in black) the threshold of $\tau$ above which (4.16) predicts the appearance of centrifugal instability. The criterion predicts the birth of centrifugal instability remarkably well for all three values of $\rho$ - including $\rho=0.33$, for which the vortex is strongly non-axisymmetric, i.e. the vortex is less concentrated and strongly deformed by the strain field.

To predict the maximum growth rate for non-axisymmetric flows using the heuristic approach, we rewrite the expression in (4.13) as:

$$
\sigma_{H}^{* 2}=4 \pi \frac{\mathrm{d} T}{\mathrm{~d} \psi} \frac{(\mathrm{d} w / \mathrm{d} \psi)^{2}-\left(1 / T^{2}\right)(\mathrm{d} \Gamma / \mathrm{d} \psi)(\mathrm{d} T / \mathrm{d} \psi)}{(\mathrm{d} T / \mathrm{d} \psi)^{2}+\left(T^{3} / \Gamma\right)(\mathrm{d} w / \mathrm{d} \psi)^{2}},
$$

where $T$ and $\Gamma$, as discussed earlier, are of the same sign. In the above expression, which is exact for axisymmetric flows, the subscript $H$ refers to a heuristic approach used.

To evaluate the validity of (4.17) for non-axisymmetric flows, in figure 7, we plot the maximum growth rate $\sigma_{H}^{*}(4.17)$ as a function of $x_{0}$ along the horizontal sections indicated by the black lines in figure 6 . Plotting the numerically calculated $\sigma^{*}$ also, we estimate the accuracy of (4.17) for $\rho=0.33,0.75$ and 0.90 . For $\rho=0.75$ and $\rho=0.90$, as shown in figure $7(b, c)$, the heuristically estimated maximum growth rate is remarkably accurate for all trajectories, including the ones far from the core of the vortices and close to the hyperbolic point. For the case of a strongly non-axisymmetric vortex $(\rho=0.33$ in figure 7a), the predictions of (4.17) are accurate for trajectories around the origin $\left(x_{0} \lesssim 1\right)$ but correspond to large errors for trajectories farther away from the origin. 
(a)

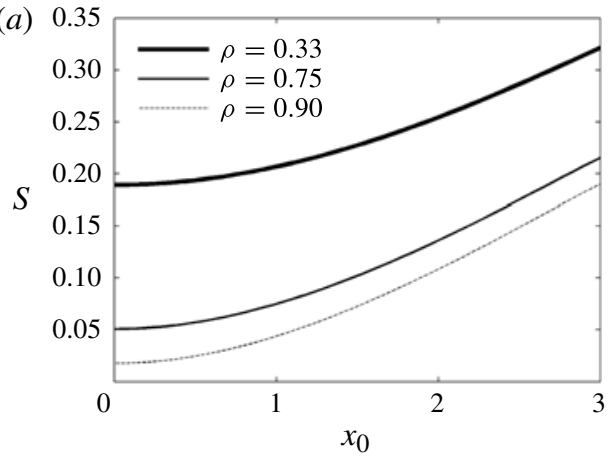

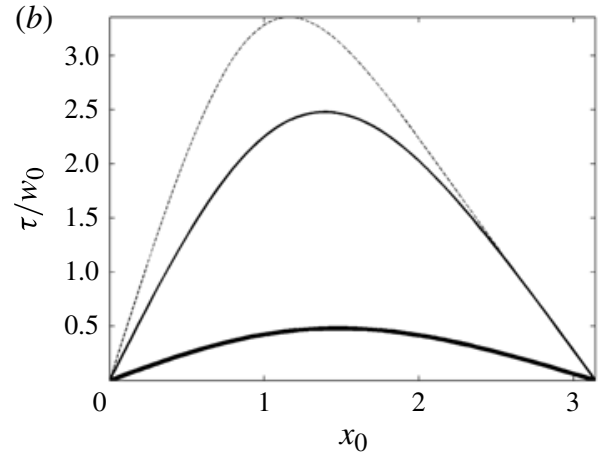

FIgURE 8. (a) The extent of non-axisymmetry, defined as $S$ in (4.18), plotted as a function of $x_{0}$ for $\rho=0.33,0.75,0.90$. (b) The variation of $\tau / w_{0}$, based on the expression in (4.20), as a function of $x_{0}$ for $\rho=0.33, m=-10$ (thick solid line); $\rho=0.75, m=-5$ (thin solid line); $\rho=0.90, m=-2$ (dashed line).

The extent of non-axisymmetry of the various streamlines in Stuart vortices is quantified using a parameter $S$, defined as:

$$
S=\frac{r_{\sigma}\left(x_{0}\right)}{\bar{r}\left(x_{0}\right)},
$$

where $r_{\sigma}$ and $\bar{r}$ are the standard deviation and mean of $r(i)=\sqrt{x(i)^{2}+y(i)^{2}}$ with $(x(i), y(i))$ being the $i$ th point on the streamline with $x(1)=x_{0}$ and $y(1)=0$. For the calculation of $S$ for Stuart vortices, every streamline is represented by 1000 points that are equispaced in terms of the distance measured along the streamline. The value of $S$ is zero for axisymmetric streamlines, and becomes progressively larger as the streamlines deviate from a circular shape.

Figure 8(a) shows the variation of $S$ as a function of $x_{0}$ for the three different values of $\rho$ considered in this paper. For a fixed value of $\rho$, the streamlines close to the origin are more axisymmetric in comparison to those close to the hyperbolic point at $x_{0}=\pi$. Furthermore, smaller values of $\rho$ correspond to larger values of $S$, the extent of non-axisymmetry. Based on the results in figure 7 , which show that the criterion in (4.17) is accurate for all streamlines for $\rho=0.75$ and $\rho=0.90$, while being inaccurate for $x_{0} \gtrsim 1$ and $\rho=0.33$, we conclude that the analytical criterion in (4.17) for centrifugal instability in non-axisymmetric vortices is valid for any streamline with $S \lesssim 0.2$; the robustness of this conclusion, however, has to be validated over a wider range of parameters for the Stuart vortices, and other non-axisymmetric vortex models.

We conclude by calculating the variation of the axial velocity parameter, $\tau$, as a function of $x_{0}$ for a typical axial velocity profile in Stuart vortices. Stuart (1967) proposed the following expression for the axial velocity $w$ :

$$
w=f(\psi)=w_{0}\left[1-(1+m \rho) \mathrm{e}^{-2 \psi}\right]^{1 / 2},
$$

where $w_{0}$ and $m$ are parameters. Upon using the expression for $\psi$ in (3.1), the expression for $\tau$ in (3.4) reduces to:

$$
\tau=w_{0} \rho \frac{1+m \rho}{1-\rho^{2}} \sin x_{0}\left[\left(1-\rho \cos x_{0}\right)^{2}-(1+m \rho)\right]^{-1 / 2} .
$$


Shown in figure $8(b)$ is the variation of $\tau / w_{0}$ with $x_{0}$ for three different combinations of $(\rho, m)$. For a given $(\rho, m), \tau$ is zero at $x_{0}=0$ and $x_{0}=\pi$, attaining a maximum for some intermediate streamline; the maximum value of $\tau$ depends on the specific values of $\rho$ and $m$. Depending on the value of $w_{0}$, it is possible to achieve any value of $\tau$ for all the streamlines in the range $0<x_{0}<\pi$. It would be insightful, however, to estimate the values of $\tau$ for various real-life flows, and hence quantify the influence of the axial flow on their stability.

\section{Conclusions}

In this paper, we have performed a local stability analysis of Stuart vortices with an axial flow. The axial flow modifies the periodicity criterion for the wavevector $\boldsymbol{k}$, a necessary condition for correspondence with the normal mode analysis. The modified periodicity criterion was derived and presented in terms of quantities that are computationally straightforward to calculate.

The elliptic and hyperbolic instabilities, that exist in Stuart vortices with no axial flow, were shown to get suppressed in the presence of a sufficiently strong axial flow. Further increase in the axial flow triggers the birth of a new branch of centrifugal instability, which was previously known only for axisymmetric vortices (Leibovich \& Stewartson 1983) from a global mode analysis. We then proposed a heuristic criterion for the onset (and corresponding growth rates) of centrifugal instability in non-axisymmetric flows, and numerically verified that the criterion makes accurate predictions for the centrifugal instability in Stuart vortices.

Further semi-analytical studies to understand the influence of an axial flow on the elliptical and hyperbolic instabilities would result in the predictions of the variations of $\tau_{E}$ and $\tau_{H}$ as functions of $\rho$ and $x_{0}$. The relevance of the local stability results presented in this paper are to be confirmed by complementary global stability analysis.

\section{Acknowledgements}

We acknowledge funding from Agence Nationale de la Recherche grant ANR 09JCJC-0108-01. M.M. benefitted from a post-doc fellowship from École Polytechnique for this work. M.M. also acknowledges support from LEGI, Grenoble, France during a part of this work. We also thank the anonymous referees for their comments, which helped improve the manuscript.

\section{Appendix}

The results presented in figures 5 and 6 correspond to the positive set of the initial wavevectors represented in (2.17) for an initial position $\left(x_{0}, 0\right)$, i.e. $\beta(t=0)=\beta_{+}^{i}$. The results are identical for the choice $\beta_{0}=\beta_{-}^{i}$ for all the initial positions on the $x$-axis for the base flow described in $\S 3$. For general initial positions, however, $\sigma$ as a function of $\theta^{i}$ depends on the branch of initial wavevectors we choose to investigate. In figure $9(a, b)$, the growth rate $\sigma$ is plotted as a function of $\bar{\theta}^{i}=\left(\theta^{i}-\theta_{\min }^{i}\right) /\left(\pi / 2-\theta_{\min }^{i}\right)$ and $\tau$ for the $\beta_{+}^{i}$ and $\beta_{-}^{i}$ branches, respectively. The initial condition $\left(x^{i}, y^{i}\right)$ for these plots is chosen such that (i) the trajectory that intersects the $x$-axis at $\left(x_{0}, 0\right)$ passes through $\left(x^{i}, y^{i}\right)$ and (ii) $y^{i}=x^{i} \tan \left(30^{\circ}\right)$. We observe noticeable differences between figure $9(a)$ and $(b)$, suggesting that the functional dependence of $\sigma$ on $\theta^{i}$ depends on the initial condition and the branch of $\beta^{i}$. The variation of $\sigma^{*}$ (the maximum $\sigma$ over the range $\theta_{\min }^{i} \leqslant \theta^{i} \leqslant \pi / 2$ across the $\beta_{+}^{i}$ and $\beta_{-}^{i}$ branches), however, does not depend on the initial position, as is evident from the fact that the calculations based on the 

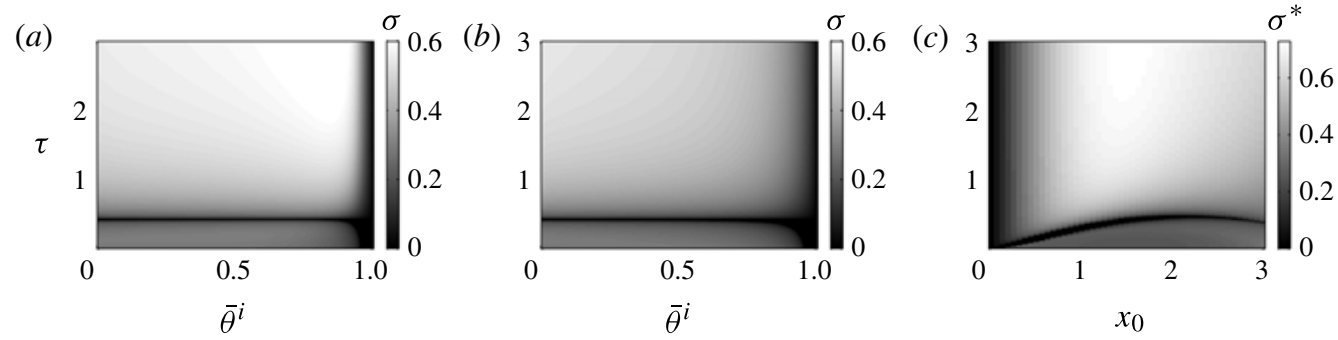

FIgURE 9. Growth rate $\sigma$ as a function of $\bar{\theta}^{i}=\left(\theta^{i}-\theta_{\min }^{i}\right) /\left(\pi / 2-\theta_{\min }^{i}\right)$ and $\tau$ for the (a) $\beta_{+}^{i}$ and $(b) \beta_{-}^{i}$ branches for $\rho=0.33$ and $x_{0}=2.69$. (c) Maximum growth rate $\sigma^{*}$ as a function of $x_{0}$ and $\tau$ for $\rho=0.33$. The initial conditions for all the plots are given by $\left(x^{i}, x^{i} \tan \left(30^{\circ}\right)\right)$ with the trajectory passing through $\left(x_{0}, 0\right)$.

initial position $\left(x_{0}, 0\right)$ (shown in figure $6 b$ ) agree quantitatively with the calculations based on the initial position $\left(x^{i}, x^{i} \tan \left(30^{\circ}\right)\right.$ ) ( shown in figure $9 c$ ).

\section{REFERENCES}

BAYly, B. J. 1986 Three-dimensional instability of elliptical flow. Phys. Rev. Lett. 57 (17), 2160-2163.

BAYLY, B. J. 1988 Three-dimensional centrifugal-type instabilities in inviscid two-dimensional flows. Phys. Fluids 31, 56-64.

Bayly, B. J., Holm, D. D. \& Lifschitz, A. 1996 Three-dimensional stability of elliptical vortex columns in external strain flows. Phil. Trans. R. Soc. Lond. A 354, 895-926.

Bender, C. M.\& Orszag, S. A. 1999 Advanced Mathematical Methods for Scientists and Engineers - Asymptotic Methods and Perturbation Theory. Springer.

Billant, P., Chomaz, J. M. \& Huerre, P. 1998 Experimental study of vortex breakdown in swirling jets. J. Fluid Mech. 376, 183-219.

Chicone, C. 2000 Ordinary Differential Equations with Applications. Springer.

Dubos, T., BARThlott, C. \& Drobinski, P. 2008 Emergence and secondary instability of Ekman layer rolls. J. Atmos. Sci. 65, 2326-2342.

EскноғF, K. S. 1984 A note on the instability of columnar vortices. J. Fluid Mech. 145, 417-421.

ECKHoff, K. S. \& Storesletten, L. 1978 A note on the stability of steady inviscid helical gas flows. J. Fluid Mech. 89, 401-411.

Friedlander, S. \& VISHIK, M. M. 1991 Instability criteria for the flow of an inviscid incompressible fluid. Phys. Rev. Lett. 66, 2204-2206.

Gallaire, F. \& Chomaz, J. M. 2003 Mode selection in swirling jet experiments: a linear stability analysis. J. Fluid Mech. 494, 223-253.

Gallaire, F., Rott, S. \& Chomaz, J. M. 2004 Experimental study of a free and forced swirling jet. Phys. Fluids 16, 2907-2917.

Gallaire, F., Ruith, M., Meiburg, E., Chomaz, J. M. \& Huerre, P. 2006 Spiral vortex breakdown as a global mode. J. Fluid Mech. 549, 71-80.

Godeferd, F. S., CAMBon, C. \& Leblanc, S. 2001 Zonal approach to centrifugal, elliptic and hyperbolic instabilities in Stuart vortices with external rotation. J. Fluid Mech. 449, 1-37.

Hall, M. G. 1972 Vortex breakdown. Annu. Rev. Fluid Mech. 4, 195-218.

HattoRi, Y. \& FukUmoto, Y. 2003 Short-wavelength stability analysis of thin vortex rings. Phys. Fluids 15 (10), 3151-3163.

Hattori, Y. \& FukUmoto, Y. 2012 Effects of axial flow on the stability of a helical vortex tube. Phys. Fluids 24, 054102; 1-15.

HATtORI, Y. \& HiJiYA, K. 2010 Short-wavelength stability analysis of Hill's vortex with/without swirl. Phys. Fluids 22, 074104; 1-8. 
HeAley, J. J. 2008 Inviscid axisymmetric absolute instability of swirling jets. J. Fluid Mech. 613, $1-33$.

Kerswell, R. R. 2002 Elliptical instability. Annu. Rev. Fluid Mech. 34, 83-113.

LACAZE, L., Birbaud, A. L. \& Le Dizès, S. 2005 Elliptic instability in a Rankine vortex with axial flow. Phys. Fluids 17, 017101; 1-5.

Leblanc, S. 1997 Stability of stagnation points in rotating flows. Phys. Fluids 9 (11), 3566-3569.

Leblanc, S. \& Le Duc, A. 2005 The unstable spectrum of swirling gas flows. J. Fluid Mech. 537, 433-442.

LE DizÈs, S. \& EloY, C. 1999 Short-wavelength instability of a vortex in a multipolar strain field. Phys. Fluids 11, 500-502.

Le Duc, A. \& Leblanc, S. 1999 A note on Rayleigh stability criterion for compressible flows. Phys. Fluids 11, 3563-3566.

Leibovich, S. 1978 The structure of vortex breakdown. Annu. Rev. Fluid Mech. 10, 221-246.

Leibovich, S. \& Stewartson, K. 1983 A sufficient condition for the instability of columnar vortices. J. Fluid Mech. 126, 335-356.

Liang, H. \& MAXWorthy, T. 2005 An experimental investigation of swirling jets. J. Fluid Mech. 525, 115-159.

Lifschitz, A. \& Hameiri, E. 1991 Local stability conditions in fluid dynamics. Phys. Fluids A 3 (11), 2644-2651.

Lifschitz, A. \& Hameiri, E. 1993 Localized instabilities of vortex rings with swirl. Commun. Pure Appl. Maths XLVI, 1379-1408.

Lifschitz, A., Suters, W. H. \& Beale, J. T. 1996 The onset of instability in exact vortex rings with swirl. J. Comput. Phys. 129, 8-29.

Loiseleux, T., Chomaz, J.-M. \& Huerre, P. 1998 The effect of swirl on jets and wakes: linear instability of the Rankine vortex with axial flow. Phys. Fluids 10, 1120-1134.

Oberleithner, K., Sieber, M., Paschereit, C. O., Petz, C., Hege, H. C., Noack, B. R. \& WYGNANSKI, I. 2011 Three-dimensional coherent structures in a swirling jet undergoing vortex breakdown: stability analysis and empirical mode construction. J. Fluid Mech. 679, 383-414.

Saffman, P. G. 1992 Vortex Dynamics. Cambridge University Press.

StUart, J. T. 1967 On finite amplitude oscillations in laminar mixing layers. J. Fluid Mech. 29, $417-440$. 